The Piecewise Linear Ballistic Accumulator 1

Running head: THE PIECEWISE LINEAR BALLISTIC ACCUMULATOR

A new framework for modeling decisions about changing information: The Piecewise

Linear Ballistic Accumulator model

\author{
William R. Holmes ${ }^{1}$ \\ Department of Physics and Astronomy, Vanderbilt University \\ Department of Mathematics, University of Melbourne, Australia \\ Jennifer S. Trueblood ${ }^{1}$ \\ Department of Psychology, Vanderbilt University \\ Department of Cognitive Sciences, University of California, Irvine
}

Andrew Heathcote

School of Medicine, University of Tasmania, Australia 
The Piecewise Linear Ballistic Accumulator 2

\begin{abstract}
In the real world, decision making processes must be able to integrate non-stationary information that changes systematically while the decision is in progress. Although theories of decision making have traditionally been applied to paradigms with stationary information, non-stationary stimuli are now of increasing theoretical interest. We developed the piecewise linear ballistic accumulator (PLBA) model to explain how decision processes are updated when a stimulus changes. The PLBA is efficient to simulate, enabling it to be fit to participant choice and response-time distribution data in a hierarchal modelling framework using a non-parametric approximate Bayesian algorithm. We applied the PLBA to a random-dot motion paradigm in which participants viewed a cloud of moving dots, where the motion switched directions midway through some trials, and were asked to determine the direction of motion. Behavioral results revealed a strong delay effect: after presentation of the initial motion direction there is a substantial time delay before the changed motion information is integrated into the decision process. Consistent with behavioral results, PLBA fits confirmed the presence of a long delay between presentation and integration of new stimulus information, but did not support increased response caution in reaction to the change. We also found the decision process was not veridical, as symmetric stimulus change had an asymmetric effect on the rate of evidence accumulation. Our results show that in this paradigm the perceptual decision process was slow to react to, and underestimated, new contrary motion information. These results support the more general utility of the PLBA model as a flexible framework for investigating decisions about changing information.
\end{abstract}

Keywords: evidence accumulation models, non-stationary stimuli, random-dot motion, hierarchal Bayesian inference 
The Piecewise Linear Ballistic Accumulator 3

\section{A new framework for modeling decisions about changing information: The Piecewise Linear Ballistic Accumulator model}

\section{Introduction}

The ability of individuals to update their decision process in the face of dynamically changing information is important in everyday decision-making. For example, consider the simple act of changing lanes on a busy highway. At first, the lane looks clear, but then a car swoops in from the other side. In order to avoid a collision, you must be able to analyse the new information and change your course of action. Although common and clearly of practical importance, such "non-stationary" decisions, where contrary pieces of information are sequentially experienced, are challenging to investigate, both empirically and theoretically.

In this paper we investigate the effect of switching perceptual evidence from favouring one choice to another during the course of the deliberation process, with the aim of developing a tractable and flexible framework to model such situations. Initial conventional analyses revealed a surprising sluggishness or delay in the way the decision process reacts to the changed perceptual (motion) information. We then developed a cognitive model to explain how evidence for each choice is accumulated in order to gain detailed insights into the causes of this delay. The model allows us to compare several explanations of the observed delay. One possibility is that, in reaction to the conflict caused by the change, participants delay their response by requiring a higher standard of evidence. Alternatively there may be a delay before the new information changes the input to the decision process. We also examined whether the input to the decision process is veridical, that is, whether it represents the true magnitude of the change. Before 
The Piecewise Linear Ballistic Accumulator 4

describing the model and reporting the experimental findings in detail we first provide a brief background on evidence accumulation models and decisions based on non-stationary or conflicting inputs.

Evidence accumulation and non-stationary decision processes

Although Ratcliff (1980) discussed the importance of changing information over three decades ago, most quantitative models of decision making have focused on "stationary" decisions, where a choice is made on the basis of fixed, unchanging information, or on information that changes randomly around a fixed central tendency (Ratcliff, 1978; Busemeyer \& Townsend, 1993; Shadlen \& Newsome, 1996; Gold \& Shadlen, 2001; Smith \& Ratcliff, 2004). Much of this work has supported the idea that decisions are based on evidence for different alternatives that is accumulated over time. A decision is made as soon as a threshold amount of accumulated evidence in favor of one of the choices is obtained. The use of stationary stimuli, and the assumption that they cause a constant rate of evidence accumulation, has made it possible to derive relatively easily computed model predictions for choices as well as the full distribution of response time (RT) for each choice. This setup has enabled models such as the drift-diffusion model (DDM) - Ratcliff's elaboration (Ratcliff, 1978; Ratcliff \& McKoon, 2008) of the simple diffusion model (Edwards, 1965) - and the linear ballistic accumulator (LBA) model (Brown \& Heathcote, 2008) - a simplification of the Leaky Competitive Accumulator (Usher \& McClelland, 2001) and Ballistic Accumulator (Brown \& Heathcote, 2005a) models - to be tested against detailed patterns of behavior across a wide range of paradigms with stationary stimuli. Accounting for such detailed findings, including the exact shape of RT distributions and the relative speed of correct and incorrect responses, has become a benchmark for models that claim to provide a general account of choice RT. The impact of non-linear and non-stationary inputs on decision-making has 
The Piecewise Linear Ballistic Accumulator 5

traditionally received less attention, but recently there has been increasing interest from a number of perspectives. One aspect concerns the effect of fluctuations on fast time scales (e.g., Insabato, Dempere-Marco, Pannunzi, Deco, \& Romo, 2014; Tsetsos, Usher, \& McClelland, 2011; Brunton, Botvinick, \& Brody, 2013), but our focus here is on the domain of slower time-scale changes. Early work in this domain examined temporal-order discrimination, where discriminative information is available only briefly, and therefore must be subsequently held in a decaying visual short-term memory (Heath, 1981). A variant of the simple diffusion model, the "tandem random walk", proposed that such discriminations could be modelled using a piecewise linear approximation, where an initial period with an estimated non-zero mean accumulation rate is followed by a period with a zero mean rate. More broadly, piecewise-linear approximations are used across the sciences to make the analysis of non-linear change tractable (e.g., Euler's method, Atkinson, 1989). As we discuss in detail below, we apply this approach to a stimulus that changes during an ongoing decision by proposing a piecewise linear ballistic accumulator (PLBA) model that has two - potentially different - pairs of estimated accumulation rates rather than just one.

More recent studies have used stimuli that change on a slow time scale in two broad ways: (1) to test assumptions made by evidence-accumulation models and (2) as of interest in their own right. Assumptions have been tested by manipulating when discriminative information occurs over the time course of stimulus presentation in the random dot motion (RDM) discrimination task (Ball \& Sekuler, 1982; Britten, Shadlen, Newsome, \& Movshon, 1992, 1993). In this task - which we also use in the present experiment - participants view a cloud of dots, some of which move randomly and some of which move coherently, and are asked to choose the dominant direction of motion. In Huk and Shadlen (2005), brief motions pulses were used to identify a neural substrate for accumulation in the lateral intraparietal area of monkeys, differentiating cells whose firing 
The Piecewise Linear Ballistic Accumulator 6

rate followed the pulse versus those that did not (see also Gold \& Shadlen, 2007). In another recent example, Winkel, Keuken, Van Maanen, Wagenmakers, and Forstmann (2014) used non-stationary RDM stimuli to test the urgency gating model (Cisek, Puskas, \& El-Murr, 2009), which attempts to replace the idea of evidence accumulation with an urgency factor that represents a linearly increasing time pressure to respond. Over the course of a two-second stimulus, a brief pulse of early evidence was followed by no evidence, the same evidence, or contradictory evidence for the remainder of the stimulus. Mean RT and error rates decreased with increases in the overall consistency of evidence. This pattern was captured by fits of a simple diffusion model with rates directly reflecting the stimulus, whereas the urgency-gating model failed because it predicted no change.

Non-stationary stimuli have also been used to investigate a key difference within the class of evidence-accumulation models: whether the accumulation process (as distinct from the evidence that drives it) is non-linear. One form of nonlinearity, which is almost universally adopted, occurs when accumulation ceases after a threshold amount of information is accumulated, so later arriving information has no influence. Several evidence accumulation models, including decision field theory (Busemeyer \& Townsend, 1993; Busemeyer \& Diederich, 2002), the leaky competitive accumulator model (Usher \& McClelland, 2001), and the ballistic accumulator (Brown \& Heathcote, 2005a), also assume accumulation can be nonlinear throughout its time course. Nonlinearity occurs due to loss of previously accumulated information (leakage), which causes a recency effect (i.e., late arriving information has more influence than earlier information). In the leaky competitive accumulator and ballistic accumulator models nonlinearity also occurs due to competition caused by lateral inhibition. Competition can cause a primacy effect (i.e., a greater influence of early information), because it allows old information to suppress the accumulation of new information. Although not as easily applied as the diffusion and LBA - because the nonlinearity makes them less mathematically tractable - these models have 
The Piecewise Linear Ballistic Accumulator 7

also been shown to provide a good account of many detailed behavioural benchmarks with stationary stimuli.

In Kiani, Hanks, and Shadlen (2008) the timing of brief motion pulses was manipulated in RDM displays and a primacy effect was interpreted as consistent with linear accumulation to a threshold. That is, later pulses were assumed to arrive after the threshold had been reached, and so had no influence on decisions. This work was revisited by Tsetsos, Gao, McClelland, and Usher (2012); they replicated the primacy effect, but also found it to weaken or even change to a recency effect when there was less time pressure on responding. One interpretation of the weakening of the primacy effect is that participants set a higher threshold as time pressure reduced, so that a greater number of later arriving pulses could influence the decision. Tsetsos et al. (2012) interpreted the results as consistent with a change in the balance of leakage and competition in the leaky competitive accumulator model, in particular, a reduction of competition as time pressure decreased.

Usher and McClelland (2001) also invoked the idea of a labile balance between leakage and competition to explain strong individual differences in the magnitude of primacy vs. recency effects. They used a sequence of 16 briefly-presented stimuli at a rate of 60 stimuli/second. On critical trials, half of the stimuli favored one response and half the other. The stimuli were randomly ordered except for a short cluster of one type at the end, and so a predominance of the other earlier in the sequence. One third of participants showed a preference for the end cluster (recency), one third against it (primacy) and one third were neutral (consistent with linear accumulation). The latter participants displayed the highest accuracy in trials where one type of stimulus predominated, which is consistent with the fact that linear accumulation makes the most efficient use of the available information. Brown and Heathcote (2005b) found that practice was associated with increasingly efficient (linear) accumulation, with an initial tendency towards recency 
The Piecewise Linear Ballistic Accumulator 8

effects associated with priming by a brief (30ms) pulse disappearing over the course of two hours of practice. This occurred even when the prime was metacontrast masked, so participants were not able to detect it; hence, the decrease in recency was unlikely to be due to a strategic reduction in the evidence threshold.

In the work just summarised, the focus was on non-linear effects in the accumulation process. It is also possible that the process of encoding sensory information has non-linear effects on the magnitude and timing of the inputs to the decision process. In the PLBA model, we use a piecewise-linear approximation, potentially with a delay, to account for the combined effects of stimulus change on the encoding and decision processes. In particular, we investigate whether a change in motion that is objectively symmetric (i.e., equal in absolute magnitude) has a veridical effect on the rate of evidence accumulation in the decision process (i.e., produces two rates that are equal in absolute magnitude), and whether any change in rate is immediate or delayed. If a veridical representation of the stimuli drives the decision process, such as was assumed by Winkel et al. (2014), one might expect that rates of evidence accumulation would exhibit the same symmetry as the objective information. Some delay, although perhaps only relatively minor, might be expected due to the time required to perform stimulus encoding.

In contrast, a longer delay and asymmetry in the form of a slower rate in response to the change, might be attributed to competition mechanisms in the decision process (e.g., Usher \& McClelland, 2001; Brown \& Heathcote, 2005a) - which has been used to explain primacy effects - or to hysteresis in the stimulus encoding process - which has been used to explain motion aftereffects (Hiris \& Blake, 1992; Anstis, Verstraten, \& Mather, 1998). It is also possible that higher-level phenomena, such as anchoring (Hogarth \& Einhorn, 1992; McKenzie, Lee, \& Chen, 2002), might also cause an asymmetry in encoding. For all of these possibilities, the piecewise-linear assumption made in the PLBA enables a computationally tractable yet comprehensive model (i.e., one that can account for RT 
The Piecewise Linear Ballistic Accumulator 9

distributions and choice probabilities) that can approximate potentially smoothly varying nonlinear effects in both encoding processes and in decision processes.

\section{Stimuli with multiple conflicting attributes}

Intrinsic interest in how the decision process deals with non-stationary inputs, and discrete switches of incoming information in particular, is reflected in several recent studies examining decisions about multi-attribute stimuli. In these cases, the cause of the change was endogenous, either through attention switching (Diederich, 1997; Diederich \& Busemeyer, 1999), or eye movements (Krajbich, Armel, \& Rangel, 2010; Krajbich \& Rangel, 2011), between conflicting attributes. These investigations suggested that looking at or attending to a particular choice alternative or attribute switches the rate at which evidence is accumulated. In Diederich and Busemeyer (2006) and Diederich (2008), this idea was extended to account for the effects of differential payoffs between choices, where accumulation was first based on the payoff information and then on the stimulus.

In both the eye-movement and payoff work, the simple diffusion model (Edwards, $1965)$ with non-stationary rates was fit to the data, but only at the level of choice probabilities and mean RT. More fine-grained tests were difficult because these non-stationary versions of the simple diffusion model are less mathematically tractable. The simple diffusion model is also known to fail benchmarks such as the relative speed of correct and error RT, which was the reason behind inclusions of extra features such as normal trial-to-trial variability in the mean accumulation rate and uniform variability in the starting point of evidence accumulation in the full DDM model (Ratcliff \& Rouder, 1998). The LBA model shares these assumptions about trial-to-trial variability, but assumes any effects of diffusive (i.e., moment-to-moment) noise can be neglected, making it particularly tractable.

Conflict effects in the Simon (Simon \& Rundell, 1967) and Flanker (Erikesn \& 
Eriksen, 1974) paradigms can also be seen as being due to non-stationarity caused by endogenous factors affecting the processing of multi-attribute stimuli. In the Simon effect a choice-irrelevant stimulus location attribute (e.g., positioning on the left vs. right of the screen) hurts performance when it is incompatible with the response associated with the stimulus's choice-relevant attribute (e.g., a button press by the left vs. right hand for red vs. green stimuli respectively). Dual-route theories of the Simon effect (e.g., Jong, Liang, \& Lauber, 1994) propose that the irrelevant position information is processed by a fast direct route, so when it is incompatible it causes initial evidence favouring the incorrect response before the later arrival of evidence favoring the correct choice from the relevant attribute. In the Flanker effect, a large display of irrelevant conflicting information flanks a smaller central display of relevant information (e.g., indicate the direction of the central arrow head in a display such as " $<<><<$ "). White, Ratcliff, and Starns (2011) and Hübner, Steinhauser, and Lehle (2010) modeled this task using a DDM fed by a non-stationary evidence source. White et al. examined a range of alternative models where the non-stationarity was caused by factors such as a discrete switch in attention from all stimuli to the central stimulus or (in the best-supported model) a gradually increasing relative weight given to the central stimulus.

Recent accounts of conflict tasks have emphasised the role of control processes that minimize the impact of interfering information. Botvinick, Braver, Barch, Carter, and Cohen (2001) proposed an influential theory whereby cognitive control was triggered by the simultaneous presence of conflicting reading and color naming information in the Stroop task. In the the Simon task, Ridderinkhof (2002) proposed a more temporally oriented theory, where conflicting information from the fast route is suppressed by a control process, but only after a delay, so its effect becomes evident as a decrease in the size of the interference effect only for slower responses.

Although we are not aware of the link being made before, a natural extension of 
these ideas is that the conflict caused by a change in a single stimulus attribute over the course of a decision might also trigger similar control mechanisms. Triggering may require awareness of the conflict or it might rely on an automatic mechanism. More automatic mechanisms would likely be mediated by effects on the rate of evidence accumulation. However, a suppression mechanism like that proposed by Botvinick et al. (2001) and Ridderinkhof (2002) may be less plausible in this context as there is not an irrelevant attribute that can be targeted separately from the relevant attribute.

Alternatively, as changes in threshold are usually assumed to be strategic, an increase in the evidence threshold in response to detection of conflict might plausibly mediate conscious control. However, threshold changes are often assumed to be slow. Hence, there may not be sufficient time after a change is detected to effect any substantial adjustment of the threshold, and at the very least any change might be expected to be delayed relative to the time at which the stimulus changes. In any case, in order to examine the issue of conscious mediation, at the end of the experiment we tested participants' ability to detect change trials and examined the relationship between individual differences in this ability and performance in the primary motion-direction classification task.

\section{Measuring and modelling the effects of a discrete exogenous change}

The goal of the present work is to provide a modelling framework within which to investigate how individuals adapt to a discrete exogenous change of information about a single attribute that occurs during the course of the decision process. Many of the investigations just reviewed used complex experimental designs where there were short exogenous changes, or where there were multiple endogenously generated changes. These characteristics make it difficult to probe the time course of the decision process and examine the influence of individual pieces of information. We use a simpler experimental 
setting to get a more detailed view of the underlying decision process; an RDM task where the direction of dot motion changed only once during the trial. For example, $15 \%$ of the dots might be moving coherently to the right at the beginning of the trial, but this switches to $15 \%$ moving coherently to the left halfway through the trial. In principle the framework we develop could account for other situations, such as changes in the magnitude rather than direction of movement, but we focus on direction change as it provides a strong manipulation.

Using our direction-determination task, we examine four questions. The first two questions address whether external changes in information result in commensurate changes in the decision process:

1) How quickly is new information integrated into the decision process?

2) Is the encoding of new information veridical?

The third and fourth questions addresses whether participants are aware of the change and react by altering their decision processing:

3) Does a participant's accuracy at detecting the change relate to its effect?

4) Do participants react to the change by changing their level of caution?

The initial period before the switch was calibrated to take around half of the average accumulation period for each individual decision maker, and the stimulus after the change remained on screen until a response was made. This setup, which made the two types of stimuli visible for extended periods of time, facilitated good estimation of the effects of each period of constant coherent motion. Given random variation in RT, there will be some fast trials where the response will only be influenced by the initial direction. Evidence-accumulation model-based analyses naturally take into account such variation. However, we also complemented our model-based analysis with conventional analysis, which divided trials into those with responses made before and after the switch. The subset of responses made after the change could at least in principle be a function of the 
new information, although this might not be the case if there is a delay before the new information affects the decision process. Responses made after the switch were further divided based on their speed in order to check for delay effects, and to examine the quantitative influence of the increasing amount of information from the direction change.

In contrast to previous studies that attempted to minimise participants' awareness of changes in the stimulus (e.g., Brown \& Heathcote, 2005b; Usher \& McClelland, 2001), our paradigm uses a change that is likely to be noticeable, although we did not explicitly inform participants about the presence of changes on some trials. This raises the question as to whether participants might strategically decide to react differently on change trials compared to trials with stationary stimuli. For example, on noticing a change they may become cautious, raising their evidence threshold. Alternatively, they may attempt to minimise the effect of the conflicting information by lowering their threshold. An extra test at the end of the experiment allowed us to assess whether participants' had the ability to detect changes. If they can detect the change and use it to react differently to change trials, one might expect individual differences in change-detection ability to correlate with performance on change trials. A more general model-based test of reactivity is provided by allowing for a different threshold on change and stationary trials. Because change detection and any subsequent threshold adjustment is likely time consuming, we also allowed for the threshold change to be delayed relative to the stimulus change.

Although direct analysis of choice and response time data can yield valuable insights, it is not by itself sufficient to fully address the proposed questions. To do so we utilise the PLBA model, which is schematically illustrated in Figure 1. In particular, our questions were addressed using model selection and latent parameter estimation based on the fits of different parameterisations ("variants") of the PLBA model. The variants are all special cases of the most general form of the PLBA model, which we now describe.

Brown and Heathcote's (2008) LBA model is a special case of the PLBA model, and 
like the LBA, in the PLBA there is one accumulator per response, which begin with evidence levels independently sampled from uniform distributions on the interval $[0, A]$. In general, $A$ may vary between accumulators and experimental conditions, but here we estimated only a single value. This estimate was around 1 in all models, and so we do not discuss it further.

The evidence in each accumulator begins to change linearly with a constant rate at some time after the stimulus first appears (see Figure 1, "encoding delay"). At some time after the change in the stimulus (see Figure 1, "rate delay") the rate changes, and linear accumulation continues at the new rate. At any point, the process can terminate because the evidence exceeds an accumulator's threshold.

The response, corresponding to the first accumulator to reach its threshold (i.e., that wins the race among accumulators), is then made after some further delay (see Figure 1, "response-production delay"). The encoding and response-production delays are estimated as a single parameter $t_{e r}$. In general, $t_{e r}$ may vary between accumulators and experimental conditions, but here we estimated only a single value. This estimate was around $0.12 \mathrm{~s}$ in all models, and so we do not discuss it further.

Linear rates of change (i.e., "drift rates") are independently sampled from normal distributions. To identify the model, we make the normalising assumption that the standard deviation of the normal distribution is a fixed value, $\sigma=1$, although this is stronger than is strictly necessary for identifiability (Donkin, Brown, \& Heathcote, 2009). We discuss differences in the rate means across accumulators before and after the stimulus changes when defining specific model variants.

The threshold, $b$, cannot be less than $A$ (so a response cannot be made until accumulation begins), and in general $b$ can vary between accumulators and experimental conditions. A difference between accumulators in $b$ causes response bias, with a lesser threshold increasing the chance of the corresponding response. Here we assume that 
responding is unbiased, so both accumulators have the same threshold, but allow for the possibility that the threshold can change at some time after the stimulus changes. This time is denoted as the "threshold delay" in Figure 1, which illustrates a case in which the threshold increases.

We propose a series of nested variants of the PLBA model that could account for how decision processes adapt to changed information. In the simplest variant we assume that the change in motion direction has no influence on decision processes. That is, decisions are entirely based on the early information and neither rates nor thresholds change during the decision process. As a consequence this variant is equivalent to the standard LBA model. Further variants, which are described in more detail after reporting the results of the experiment, take advantage of various aspects of the the extra flexibility that differentiates the PLBA from the LBA in order to determine which aspect(s) best account for the data.

Many researchers using non-stationary versions of the diffusion model, where drift rates are allowed to change during the trial, only fit choice probabilities and mean RTs, rather than the full distribution of RTs. However fitting statistics of RT distributions (such as the mean or quantiles) can lead to drastically different parameter estimates than fits to the full distribution (Turner \& Sederberg, 2014). We used a hierarchal Bayesian algorithm to fit each PLBA variant to all participants' data simultaneously, providing an account of the choices made and the full distribution of response times at both the individual and population levels. Bayesian methods usually rely on the availability of an expression for the likelihood of each data point (i.e., choice and associated RT). A likelihood is available for the standard LBA in terms of relatively quickly computed functions like normal densities and cumulative densities (Brown \& Heathcote, 2008). This is not the case for the PLBA, and although it is possible to compute the PLBA likelihood by numerical integration (see Appendix A), we found this to be too slow, at least using 
The Piecewise Linear Ballistic Accumulator 16

standard methods. Instead we rely on a variant Holmes (2015) of an "Approximate Bayesian Computation" (ABC) algorithm originally developed in Turner and Sederberg (2014), which requires only simulation of choices and RTs from the model. Details of its implementation are described in Appendix B and in Holmes (2015). Here, we provide a brief overview.

Numerous ABC algorithms have been developed to deal with models lacking an easily computed likelihood. Many rely on summary statistics to represent critical properties of the model and data being considered. However, this approach suffers from a number of issues, the most important of which is the requirement that a sufficiency condition on the summary statistics must be met, which is rarely possible to confirm. We instead use a combination of simulation and non-parametric density estimation to provide an approximation to the underlying likelihood that is similar to, but more computationally efficient, than the method proposed by Turner and Sederberg (2014). This is coupled to a Markov-Chain Monte Carlo (MCMC) procedure to estimate the model's posterior, with some additions to the MCMC algorithm to efficiently deal with the effects of simulation error (i.e., variation from one simulation sample to the next) in the likelihood approximation.

Even with all of the efficiencies of this method, fitting was very computationally demanding. The key to making fitting viable is that it is highly efficient to simulate ballistic models, and the PLBA model in particular. This is because, to evaluate the choice and associated RT for a trial requires, for each accumulator, only a single sample from the uniform distribution, two samples from the normal distribution, and then some simple linear calculations based on these samples and threshold and delay parameters. In contrast, models with moment-to-moment variability in accumulation rates, such as the DDM, require fine-grained Euler methods using a separate normal sample for at least each 10 milliseconds of decision time (Brown, Ratcliff, \& Smith, 2006). Similarly fine-grained 
approximation methods for solving differential equations are required for non-linear models like the Ballistic Accumulator model (Brown \& Heathcote, 2005b), and both types of approximation are required for the Leaky Competitive Accumulator model (Usher \& McClelland, 2001). The procedures used with the PLBA could be applied to these other models, but would require orders of magnitude more computational time.

In the following, we first describe our experiment and describe the results of analyses that do not rely on modeling. We then describe the variants of the PLBA that we tested, their hierarchal instantiation, and our model-based results.

\section{Experiment}

The experiment was constituted of two types of tasks: a primary RDM direction determination task, which occurred over the first 18 blocks of the experiment, and a secondary switch (e.g. change of stimulus) detection task, which occurred in the last two blocks of trials. The RDM task required participants to decide whether a cloud of dots appears to move to the left or right. There were two types of trials which were randomly mixed together: stationary trials, where the direction of motion remained constant (e.g., right for the full duration of the trial), and switch trials where the direction of motion reversed within the trial (i.e., right followed by left or vice versa). On switch trials, the change in direction occurred at a predetermined "switch time", which was adapted during the experiment based on previous RTs in order to accommodate changes due to factors like practice and fatigue. After the completion of the primary task, two additional switch-detection blocks tested whether participants could detect changes in dot motion.

\section{Material and methods}

Participants. In total, 47 University of California Irvine students (40 female, 44 right handed) completed the study for course credit. All participants gave informed consent to participate. Three participants were excluded from the data analyses due to a 
computer error. An additional 13 participants were excluded because of low accuracy after three initial practice blocks (i.e., less than $70 \%$ correct on the stationary trials in blocks 4-18). These participants were most likely not engaged throughout the task. ${ }^{2}$

Procedure. Stimuli were clouds composed of 40 white dots contained within a 150-pixel diameter area on a black background. Each dot consisted of a 9 pixel rectangle with edges smoothed through anti-aliasing to appear circular. The dot positions were updated at 15 frames per second. A subset of dots moved in a coherent direction while the remaining appeared in locations that were randomly selected on each frame. The coherent set of dots moved at a rate of 90 pixels per second from their original location until reaching the edge of the 150-pixel diameter area. On the next frame they were repositioned at a randomly chosen location and moved as before on subsequent frames.

At the beginning of the experiment, participants were instructed that they would see a group of moving dots and were asked to decide if the dots are mostly moving to the left or the right. They were told "you will enter your choices by pressing the ' $\mathrm{z}$ ' key if you think the dots are mainly moving to the left and the '/' key if you think the dots are mainly moving to the right." Participants were asked to place the index finger of their left hand of the ' $\mathrm{z}$ ' key and the index finger of their right hand on the '/' key throughout the experiment. They were also told that they would complete many blocks of trials, that some trials would be harder than others and that they would sometimes receive feedback about their responses. Participants were asked to respond to the best of their ability as quickly as possible, but were not told that the dots could possibly switch directions during some trials.

At the beginning of each trial, they viewed a fixation cross for $250 \mathrm{~ms}$, then there was a blank screen for $250 \mathrm{~ms}$, followed by the stimulus. Participants had up to $2 \mathrm{~s}$ to view the stimulus and give a response or the trial terminated by itself and a non-response was recorded. Otherwise, the trial terminated immediately after a response was made. The 
fixation cross for the next trial appeared immediately after the termination of the previous trial.

The experiment took approximately half an hour to complete, during which participants completed 20 blocks of trials. The first block contained 20 practice trials where $50 \%$ of the dots moved coherently and participants received feedback. This feedback delayed trial termination, and consisted of either "correct" or "wrong" displayed for 500 ms, followed by a blank screen for 250ms. In blocks 2-18, participants did not receive feedback. The second block contained 20 additional practice trials where $25 \%$ of the dots moved coherently. In the third block, participants completed 72 trials, which were composed of 24 trials each at the $20 \%, 15 \%$, and $10 \%$ coherence levels. At the conclusion of the third block, one of the three coherence levels was selected for the remainder of the experiment using the following algorithm to account for individual differences in motion detection ability so that performance was away from floor and ceiling:

1) If the participant's accuracy was exactly $75 \%$ for a specific coherence level, then this coherence level was selected. If more than one coherence level achieved $75 \%$ accuracy, then the lowest coherence level was selected.

2) If no coherence level achieved accuracy of exactly $75 \%$, then the coherence level with accuracy higher and closest to $75 \%$ was selected. If there was a tie between coherence levels, then the lowest one was selected.

3) If none of the coherence levels achieved $75 \%$ accuracy, then the largest coherence level (i.e., 20\%) was selected.

Blocks 4-18 each contained 72 trials with the coherence level selected at the end of block 3. Half of the trials in each block were switch trials, where the dot motion switched directions during the trial (e.g., left followed by right). The other half of the trials were stationary, where the dot motion did not change. The two trial types were randomly ordered. On switch trials, the change in direction occurred at the switch time, which was 
based on previous RTs in the experiment. In block 4, switch time was set to the median RT of block 3 minus 150ms to account for response-production time. In block 5, switch time was set to the median RT of blocks 3 and 4 minus $150 \mathrm{~ms}$. In block 6 , switch time was set to the median RT of blocks 3,4 , and 5 minus $150 \mathrm{~ms}$. For blocks $7-18$, switch time was set similarly to block 6 by using response times from the previous three blocks.

Blocks 19 and 20 tested whether participants could detect changes in dot motion. During these blocks, they were instructed to only respond if the dot motion changed directions. They were told to withhold responses if the dot motion was constant. Block 19 contained 16 practice trials with feedback. At the end of each trial, participants saw a message "correct" or "wrong" displayed for 500ms. Block 20 contained 72 trials with no feedback. In both blocks, half of the trials were switch trials and the other half were stationary, randomly ordered. The switch time for these blocks was determined in the same manner as blocks 6-18, and the coherence level was the same as blocks 4-18.

Results

Participants were evenly distributed among the three coherence levels: 10 with $10 \%$ coherence, 11 with $15 \%$ coherence, and 10 with $20 \%$ coherence. Trials with very short RTs (less than 250ms) were removed from all further analyses. On average less than $5 \%$ of trials were removed using this criterion.

We analysed accuracy on stationary and switch trials in the primary direction-determination task (i.e., blocks 4-18) by dividing the stationary trials into two categories - those with RTs before the switch time for switch trials in the same block, and those with RTs after the switch time. This allowed us to directly compare responses before and after the switch time for stationary and switch trials. These four trial types were further divided into quartiles (q1-q4) based on RTs.

Figure 2a compares the proportion of correct responses before the switch time for 
stationary and switch trials across RT quartiles. The correct response was defined relative to the first direction of motion on switch trials. A 4 (quartile) x 2 (trial type) analysis of variance yielded a main effect for quartile, $\mathrm{F}(3,240)=31.36, \mathrm{p}<.001$, showing an increase in accuracy with RT. The main effect of trial type was non-significant, $\mathrm{F}(1,240)$ $=0.009, \mathrm{p}=0.93$, showing no difference in accuracy for stationary and switch trials before the switch time. The interaction of quartile and trial type was also non-significant, $\mathrm{F}(3$, $240)=0.26, \mathrm{p}=0.86$. A Tukey test showed, at $\mathrm{p}<.05$, that the proportion of correct responses for both stationary and switch trials was significantly lower for q1 (stationary trials: $\mathrm{M}=0.68, \mathrm{SD}=0.02$; switch trials: $\mathrm{M}=0.67, \mathrm{SD}=0.2$ ) than for $\mathrm{q} 2$ (stationary trials: $\mathrm{M}=0.80, \mathrm{SD}=0.02$; switch trials: $\mathrm{M}=0.82, \mathrm{SD}=0.2$ ), $\mathrm{q} 3$ (stationary trials: $\mathrm{M}$ $=0.86, \mathrm{SD}=0.02 ;$ switch trials: $\mathrm{M}=0.86, \mathrm{SD}=0.2$ ), and $\mathrm{q} 4$ (stationary trials: $\mathrm{M}=$ $0.89, \mathrm{SD}=0.02 ;$ switch trials: $\mathrm{M}=0.88, \mathrm{SD}=0.2)$. The differences between $\mathrm{q} 2, \mathrm{q} 3$, and q4 were not significant. Thus, even though accuracy did improve with RT, this improvement only occurred between the first and second quartiles of RT.

Figure $2 \mathrm{~b}$ compares the proportion of correct responses after switch time for stationary and switch trials across RT quartiles. For these switch trials we defined a correct response relative to the new direction of motion. For example, in a trial where the dots first moved to the left and then the right, the correct response after the switch time is right. A 4 (quartile) x 2 (trial type) analysis of variance yielded a main effect for quartile, $\mathrm{F}(3,240)=23.0, \mathrm{p}<.001$. The main effect for trial type was significant, $\mathrm{F}(1,240)=$ $2230.1, \mathrm{p}<.001$, with much higher accuracy for stationary trials than switch trials after the switch time. The interaction effect was also significant, $\mathrm{F}(3,240)=69.68, \mathrm{p}<.001$.

The relationship between accuracy and RT quartiles after the switch time differs between stationary and switch trials. A post hoc Tukey test showed, at $\mathrm{p}<.05$, that the proportion of correct responses for switch trials was significantly higher for q4 $(\mathrm{M}=0.49$, $\mathrm{SD}=0.02)$ than for $\mathrm{q} 1(\mathrm{M}=0.21, \mathrm{SD}=0.02), \mathrm{q} 2(\mathrm{M}=0.19, \mathrm{SD}=0.02)$, and $\mathrm{q} 3(\mathrm{M}=$ 
$0.22, \mathrm{SD}=0.02)$. The remaining quartile comparisons for switch trials were not significant, indicating that the effect of the change in motion direction on accuracy was substantially delayed.

Unlike switch trials, accuracy decreased with increased RT for stationary trials after the switch time. A Tukey test showed that the proportion of correct responses for stationary trials was significantly higher for $\mathrm{q} 2(\mathrm{M}=0.82, \mathrm{SD}=0.02)$ and $\mathrm{q} 3(\mathrm{M}=0.80$, $\mathrm{SD}=0.02)$ than $\mathrm{q} 4(\mathrm{M}=0.73, \mathrm{SD}=0.02)$. This indicates the presence of slow errors, which often occur in difficult tasks (Luce, 1986; Ratcliff \& Rouder, 1998). The remaining quartile comparisons for stationary trials were not significant.

Figure 2c compares the proportion of correct responses after the switch time for switch trials to the proportion of error responses after the switch time for stationary trials. This allows us to examine whether correct responses after the switch time for switch trials were above chance accuracy. A 4 (quartile) x 2 (trial type) analysis of variance yielded a main effect for quartile, $\mathrm{F}(3,240)=69.78, \mathrm{p}<.001$. The main effect for trial type was significant, $\mathrm{F}(1,240)=32.00, \mathrm{p}<.001$, indicating there was a difference between correct responses for switch trials and error responses for stationary trials. The interaction effect was also significant, $\mathrm{F}(3,240)=23.00, \mathrm{p}<.001$.

A post hoc Tukey test showed, at $\mathrm{p}<.05$, that the proportion of correct responses for switch trials in $\mathrm{q} 4(\mathrm{M}=0.49, \mathrm{SD}=0.02)$ was significantly higher than the proportion of error responses for stationary trials in $\mathrm{q} 4(\mathrm{M}=0.27, \mathrm{SD}=0.02)$. Corresponding comparisons for q1-q3 were not significant. This suggests that decisions made after the switch time for switch trials for RT quartiles q1-q3 are most likely based on the perceptual evidence presented before the switch time. However, decisions with long RTs (i.e., those in q4) appear to be influenced by the perceptual evidence presented after the switch time.

We also analysed mean RTs for correct responses and for incorrect responses from trials before and after the switch time. There was no significant difference in mean RTs 
for correct choices on stationary and switch trials before the switch time, $\mathrm{t}(30)=0.23, \mathrm{p}=$ 0.82. Likewise, there was no significant difference in mean RTs for incorrect choices on stationary and switch trials before the switch time, $\mathrm{t}(30)=0.38, \mathrm{p}=0.71$. However, after the switch time, mean RTs for correct choices on switch trials were significantly longer than mean RTs for correct choices on stationary trials $(\mathrm{t}(30)=8.32, \mathrm{p}<0.001)$. In contrast, mean RTs for incorrect choices on switch trials were significantly shorter than mean RTs for incorrect choices on stationary trials after the switch time, $\mathrm{t}(30)=6.98, \mathrm{p}$ $<0.001$. These differences in mean RTs after the switch time result from a high error rate on switch trials in q1-q3, which decreases in q4.

The average hit rate in the change-detection task (i.e., block 20) was $65 \%$, significantly higher than chance, $\mathrm{t}(30)=5.42, \mathrm{p}<0.0001$, suggesting that participants could detect switches in direction, at least on some trials. Hits occurred on average 826 ms after the switch in motion direction. The average false alarm rate was also high at $42 \%$. Similar to hits, false alarms occurred on average $850 \mathrm{~ms}$ after the switch time. The correlation between detection hit rates and accuracy on switch direction-determination trials (i.e., blocks 4-18) was weak and far from significant, $r=0.18, \mathrm{p}=0.34$. This suggests that ability to detect change did not affect responding on switch trials. The correlation between detection hit rates and direction-determination accuracy on stationary trials was larger, $\mathrm{r}=0.36, \mathrm{p}=0.05$

\section{Discussion}

The results show that changes in dot motion can influence choice behavior, but this influence is slow to manifest itself. Only responses with long RTs (i.e., those in the fourth quartile of responses made after the switch time) are impacted by the changed information. Responses with shorter RTs are mainly based on the perceptual information presented early in the trial. Further, although participants can detect changes in dot 
motion, this ability does not appear to be related to performance on switch trials of the direction-determination task.

The results of our analyses so far address our first question - indicating that the effect of the change is quite delayed - and our third question - indicating that being able to detect the change does not appear to modulate the effect of the change. However, they cannot address our second and fourth questions. In order to address all four questions, we now report the results of our model-based analysis after first describing the eight model variants that we fit.

\section{PLBA Modeling}

We consider a sequence of eight models that differ in both the manner in which the evidence accumulation process is updated and whether response thresholds are adjusted upon the change of stimuli. The simplest "null" (i.e., standard LBA) model assumes the change of information has no affect on the accumulation process and that neither the drift rates nor the response threshold change after the switch in motion direction. The behavioral results reported so far suggest this is not the case; however, we include the simplest model as a baseline allowing us to confirm that more complex models can robustly detect the influence of the changed information. We also examine an extension of the null model where drift rates are allowed to change in response to the change of information, but the distributional properties of the drift rates do not change.

The remaining six models (see Table 1) all assume that drift rates change at some point after the stimulus changes. Three of these models $(1 \mathrm{~s}, 2 \mathrm{~s}$ and $3 \mathrm{~s})$ assume this change is symmetric. That is, rates after the change have the same absolute magnitude as the rates before the change. The other three models (1f, $2 \mathrm{f}$ and $3 \mathrm{f}$ ) freely estimate rates before and after the change. A comparison between these two classes of models answers the question, is the accumulation process veridical? Our stimuli changed symmetrically, so 
that after the switch, the direction of motion is flipped but with the same coherence. If the accumulation process is veridical, we would expect the change in the underlying drift rates to be symmetric as well.

Differences within each of the sets of three models are a result of manipulating the presence or absence of two factors: a delay in the onset of the drift rate change and a difference in the evidence threshold between stationary and switch trials. In the first variant (1s and 1f) a delayed rate change is possible, but there is no threshold change. In the second variant (2s and $2 \mathrm{f})$ a delayed threshold difference is possible, but there is no delay in the rate change. The final variant (3s and 3f) can have a delayed rate change, as in the first variant, and can also have a threshold change, but without a delay. We chose not to include the threshold delay in the third variant, as in the second variant, because the threshold delay was always estimated so long that the threshold change had a negligible effect on performance, and this was also the case when we allowed both delayed rate and threshold changes. ${ }^{3}$ By forcing no threshold delay in the third variant, we were able to ensure that estimates of the changed threshold were influenced by the data.

Before describing each variant in detail, we define some terminology. The terms "correct" and "incorrect" (with respect to the response made to a stimulus) or "matched" and "mis-matched" (with respect to the relationship between stimulus and accumulator, i.e., a left stimulus matches the left-response accumulator and mismatches the right-response accumulator) are somewhat ambiguous in switch trials. Hence, we identify choice "C1" with the correct response relative to the pre-switch stimulus direction (i.e., the pre-switch correct choice) and choice "C2" to be the alternative (i.e., the post-switch correct choice). For example, in a trial that presents left followed by right motion, the pre-switch correct choice $(\mathrm{C} 1)$ would refer to left and the post-switch correct choice $(\mathrm{C} 2)$ to right. Similarly, a stationary trial with persistent motion to the left, the pre-switch correct choice $(\mathrm{C} 1)$ would refer to left and the alternative choice $(\mathrm{C} 2)$ to right. We further 
The Piecewise Linear Ballistic Accumulator 26

aggregate over symmetric trial types, which is typical for modelling RDM tasks. So for stationary trials we aggregate results for left and right motion stimuli, and for switch trials we aggregate results for left-then-right and right-then-left motion stimuli. Hence, the pre-switch correct choice $(\mathrm{C} 1$, and a sub-script "1" for associated parameters) always refers to the initial direction and the post-switch correct choice $(\mathrm{C} 2$, and sub-script "2" for associated parameters) to the other direction. Note that in figures, black indicates results associated with the choice pre-switch correct choice (C1) and grey with post-switch correct choice $(\mathrm{C} 2)$.

Model 0. In order to determine if the change of information influences evidence evidence accumulation or thresholds, we first assume a null model where the change influences neither. Consider first the accumulation process on a stationary trial. In this framework, accumulation is characterised by a start point $x_{0}$ for each accumulator, an evidence threshold $\left(b_{1}\right)$, accumulation rates $v_{1}, v_{2}$ for pre-switch correct $(\mathrm{C} 1)$ and alternative $(\mathrm{C} 2)$ choices respectively, and the non-decision time for stimulus encoding and response production, $t_{e r}$. The start point and drift rates are assumed to vary among trials and across participants, and to be distributed according to:

$$
x_{0}=U(0, A), \quad v_{1} \sim N\left(\mu_{v 1}, \sigma\right), \quad v_{2} \sim N\left(\mu_{v 2}, \sigma\right),
$$

where $U$ and $N$ refer to uniform and normal distributions respectively. Since all conditions but the switch time remain constant across trials, the threshold $(b)$ and non-decision time $\left(t_{e r}\right)$ are assumed to remain constant across trials, but vary among participants. The accumulation process for each participant (indexed $p=1 \ldots P$ ) is described by a vector of five parameters $\theta^{p}=\left(b_{1}^{p}, A^{p}, \mu_{v 1}^{p}, \mu_{v 2}^{p}, t_{e r}^{p}\right)$. In all future model variants, we will assume the pre-switch evidence accumulation process is governed by this process. In this null model, we will further assume that the change of information does not influence the evidence 
accumulation processes, so that these parameters fully define Model 0.

Model 00. We also test an extension of the null model where it is assumed that a change of evidence accumulation rates $\left(w_{1}, w_{2}\right)$ occurs after the change, but the new information does not influence accumulation so that those rates are drawn from the same distributions

$$
w_{1} \sim N\left(\mu_{v 1}, \sigma\right), \quad w_{2} \sim N\left(\mu_{v 2}, \sigma\right),
$$

This model will be labeled "Model 00". It is possible that trials with slow RTs will be associated with a lower pre-switch drift, and also a lower accumulated difference between the two alternatives, making the choice more prone to a reversal in the post-switch period where the new drifts are sampled, and providing a parsimonious explanation of the accuracy difference between stationary and switch trials for the slowest quartile of RTs. We used Model 00 to test this possibility.

Models $1 \mathrm{~s}$ and $1 \mathrm{f}$. In these and all subsequent model variants, we assume that the presentation of new information leads to a change in the accumulation process. In all cases - based on the observation that the new information does influence choice probabilities - we assume that the change of information leads to a change in the evidence accumulation rate so that after the change of information, $v_{1} \rightarrow w_{1}$ (for the pre-switch correct accumulator) and $v_{2} \rightarrow w_{2}$ (for the post-switch correct accumulator). This change can take one of two forms, symmetric or free. In the symmetric case, it is assumed that the drift rates for the choice pre-switch correct $(\mathrm{C} 1)$ and post-switch correct $(\mathrm{C} 2)$ accumulators before and after the change are drawn from the same distributions as before the change:

$$
v_{1}, w_{2} \sim N\left(\mu_{v 1}, \sigma\right), \quad v_{2}, w_{1} \sim N\left(\mu_{v 2}, \sigma\right)
$$


Note, this is not a simple flipping of the drift rates, as although the pairs of rates are drawn from the same distribution, the individual values that are sampled vary randomly and independently. However the symmetry does reflect the symmetry of the presented stimuli. Alternatively, the switch can be free, so that pre and post change drift rates are unrelated, so:

$$
w_{1} \sim N\left(\mu_{w 1}, \sigma\right), \quad w_{2} \sim N\left(\mu_{w 2}, \sigma\right)
$$

We will reference models with either the postfix " $s$ " or " $f$ " to indicate the type of drift rate change assumed. So, for example, Model $1 s$ refers to a variant of Model 1 with a symmetric change of drift rates.

In Model 1, we assume there is a delay between the presentation and integration of new information, and include a delay parameter $\left(t_{\text {rate }}\right)$, so that if the change of information occurs at $t_{0}$ experimentally, the change in drift rates occurs at $t_{0}+t_{\text {rate }}$. In the symmetric case, each participant is characterized by a vector of six parameters $\theta^{p}=\left(b_{1}^{p}, A^{p}, \mu_{v 1}^{p}, \mu_{v 2}^{p}, t_{e r}^{p}, t_{r a t e}^{p}\right)$. In the free case, each participant is described by a vector of eight parameters $\theta^{p}=\left(b_{1}^{p}, A^{p}, \mu_{v 1}^{p}, \mu_{v 2}^{p}, \mu_{w 1}^{p}, \mu_{w 2}^{p}, t_{e r}^{p}, t_{r a t e}^{p}\right)$.

Models 2s and 2f. As with Model 1, we again assume that a change of stimulus leads to a change of drift rates, again with both symmetric and free variants. In contrast to Model 1, however, in Model 2 we assume the change in rates occurs instantaneously. Model 2 also differs from Model 1 in that it allows the evidence threshold to differ before and after the change. Rather than assuming this change occurs instantaneously, we account for the possibility that there is some delay in this adjustment, and include a delay parameter $t_{\text {thresh }}$. So, if the stimulus changes at time $t_{0}$, the response threshold is $b_{1}$ for $t<t_{0}+t_{\text {thresh }}$ and $b_{2}$ for $t>t_{0}+t_{\text {thresh }}$. In the symmetric case, each participant is characterized by a vector of seven parameters $\theta^{p}=\left(b_{1}^{p}, b_{2}^{p}, A^{p}, \mu_{v 1}^{p}, \mu_{v 2}^{p}, t_{e r}^{p}, t_{t h r e s h}^{p}\right)$. In the free case, each participant is described by a vector of nine parameters 
$\theta^{p}=\left(b_{1}^{p}, b_{2}^{p}, A^{p}, \mu_{v 1}^{p}, \mu_{v 2}^{p}, \mu_{w 1}^{p}, \mu_{w 2}^{p}, t_{e r}^{p}, t_{t h r e s h}^{p}\right)$.

Models 3s and 3f. Model 3, includes both the delayed rate effect, as well as a threshold change, but without a delay. In the symmetric case, each participant is characterized by a vector of seven parameters $\theta^{p}=\left(b_{1}^{p}, b_{2}^{p}, A^{p}, \mu_{v 1}^{p}, \mu_{v 2}^{p}, t_{e r}^{p}, t_{\text {rate }}^{p}\right)$. In the free case, each participant is described by a vector of nine parameters $\theta^{p}=\left(b_{1}^{p}, b_{2}^{p}, A^{p}, \mu_{v 1}^{p}, \mu_{v 2}^{p}, \mu_{w 1}^{p}, \mu_{w 2}^{p}, t_{e r}^{p}, t_{r a t e}^{p}\right)$. Thus, Models 2 and 3 have the same number of parameters.

\section{Hierarchal model extension}

We extend each of these individual level variants to a hierarchal model in order to compare models at the population level. We specify the following priors for the individual level parameters

$$
\begin{aligned}
b_{1}^{p}, b_{2}^{p} & \sim T N\left(\mu_{b}, \sigma_{b}, 0, \infty\right), \\
A^{p} & \sim T N\left(\mu_{A}, \sigma_{A}, 0, \infty\right) \cdot \chi_{\left[0, b_{s}\right]}, \\
\mu_{v 1, v 2, w 1, w 2}^{p} & \sim N\left(\mu_{V 1, V 2, W 1, W 2}, \sigma_{V 1, V 2, W 1, W 2}\right), \\
t_{\text {rate }}^{p} & \sim T N\left(\mu_{\text {rate }}, \sigma_{\text {rate }}, 0, \infty\right), \\
t_{\text {thresh }}^{p} & \sim T N\left(\mu_{\text {thresh }}, \sigma_{\text {thresh }}, 0, \infty\right), \\
t_{\text {er }}^{p} & \sim T N\left(\mu_{\text {er }}, \sigma_{\text {er }}, 0, \infty\right),
\end{aligned}
$$

where $T N(a, b, c, d)$ refers to the normal distribution with mean $a$ and standard deviation $b$ truncated to the interval $[c, d]$. Note that we do allow drift rates to be negative to account for the possible feedforward suppression of one alternative by the other, and so do not truncate drift rate distributions. Further, we incorporate the condition $A^{p}<b^{p}$ explicitly in the prior; $\chi_{[a, b]}$ denotes the indicator function that is 1 on the interval $[a, b]$ and 0 elsewhere. 
We further specify the following mildly informative priors for the hyper mean and standard deviation parameters

$$
\begin{aligned}
\mu_{b_{1}, b_{2}, A} & \sim T N(1,0.5,0, \infty), & \sigma_{b_{1}, b_{2}, A} & \sim \Gamma(1,1), \\
\mu_{V 1, V 2, W 1, W 2} & \sim N(3,1.5), & \sigma_{V 1, V 2, V 3, V 4} & \sim \Gamma(1,1), \\
\mu_{\text {rate }, \text { thresh }} & \sim T N(0.6,0.3,0,3), & \sigma_{\text {rate,thresh }} & \sim \Gamma(1,0.5), \\
\mu_{\text {er }} & \sim T N(0.25,0.25,0, \infty), & \sigma_{e r} & \sim \Gamma(1,0.5) .
\end{aligned}
$$

These priors are nearly the same as those in the Turner, Sederberg, Brown, and Steyvers (2013), but with a few adjustments to more accurately reflect the approximate size of various parameters. Note that the upper bound of the truncated normal for $\mu_{\text {rate }}$ was chosen for numerical reasons. As discussed in the results, estimates of the parameter $t_{\text {thresh }}$ were beyond maximum response times, creating numerical inefficiencies. This truncation keeps the value of this parameter from growth without bound, but is significantly larger than any plausible estimate.

The foregoing describes the hierarchal model for the free switch model with a fixed threshold. For the simpler symmetric switch model, the priors and hyper-priors for $w_{1,2}$ are unused and for the simplest LBA model, the priors for $t_{\text {rate }}$ and $t_{\text {thresh }}$ are also unused. In cases where multiple boundaries are considered, $b_{1,2}$ are assigned the same priors but the additional constraint that $b_{2}>b_{1}$ is applied. Conceptually, participants might also reduce the threshold, but that did not appear to be the case here, and enforcing this constraint produced better behaved estimation.

\section{Model Selection Results}

Table 1 shows the Deviance Information Criterion (DIC) (Spiegelhalter, Best, Carlin, \& Van Der Linde, 2002) values for each of the eight models fit to the data. Models 
with lower DIC values are preferred, and an advantage of 10 or more indicates a strong advantage. The first thing we note is that the non-switch model (Model 0) performs the worst of all models. That is, even though it has an advantage in simplicity over the other models, with only five parameters per participant, that advantage is lost because of its poor fit. This strongly suggests the change of information in the direction determination task is having an effect on the evidence-accumulation process. Model 00 also performs quite poorly, suggesting that taking a new rate sample post-switch from the pre-switch rate distributions cannot account for our data.

It is also clear that for each of the three model types with either a drift rate delay, a delayed threshold change, or both, the free switch version is strongly preferred, with DIC advantages that range from 15 to 35 . This clearly indicates that the change of drift rates is not symmetric. We discuss this finding further below in reference to Figure 3, which demonstrates that - in agreement with the DIC results - when freely estimated, population posterior drift rate estimates displayed a marked asymmetry.

Table 1 also clearly shows that the delayed drift rate change alone provides a substantially better account of the the data than does a delayed change in the response threshold alone. This is evident in a substantially better DIC for Model 1 (drift-delay alone) than Model 2 (threshold-delay alone), regardless of whether drift rates are assumed symmetric (an advantage of 50) or not (an advantage of 40). Adding an immediate threshold change to the drift-delay model (i.e., Model 1 vs. Model 3) produces a relatively modest improvement in DIC. When drift rates are assumed symmetric the improvement is larger (14), but this may just be overfitting in an attempt to compensate for the overall much worse fit under the symmetric assumption. When drift rates are allowed to be asymmetric the improvement is small (4), and as we discuss next, posterior parameter estimates indicate that the magnitude of the threshold change was very small. 
The Piecewise Linear Ballistic Accumulator 32

\section{Parameters Estimates}

Figure 3 shows the posterior hyper-mean estimate distributions for two model variants that both allow asymmetric drift rates but only one other effect of the switch: either a delay in the rate change (Model 1f, left column) or a delayed change in the threshold (Model 2f, right column). The central tendencies of these distributions provide an estimate of the population mean value for each parameter, and their spread represents uncertainty about these values. We first focus on the two pairs of drift-rate estimates for each model shown in rows two and three of Figure 3.

For both switch trials before the switch and the entire time course of stationary trials, the second row shows the rates for the accumulators $\left(\mu_{V 1}\right.$ and $\left.\mu_{V 2}\right)$. Because these parameters are constrained by all of the stationary trial data as well as switch trials where responses occur before the switch, their distributions are tightly peaked, indicating relatively little estimation uncertainty. Accuracy in responding is determined to a large degree by the differential between the rates, as the accumulator corresponding to the correct choice (i.e., $\left.\mu_{V 1}\right)$ is more likely to win the race as its rate increases relative to the accumulator corresponding to the incorrect choice (i.e, $\left.\mu_{V 2}\right)$. At the aggregated-model level this differential can be measured by the difference between the average posterior mean estimates, $\bar{\mu}_{V 1}-\bar{\mu}_{V 2}$. As Figure 3 shows, this measure is virtually identical for both models, with a value of 1.3 .

Distributions for the drift rates $\left(\mu_{W 1}\right.$ and $\mu_{W 2}$ in the third row) after the change of motion however indicate substantive differences between the two models. Uncertainty in these estimates is somewhat greater as they are constrained by less data, only those switch trials where responses occur after the change. The locations of these distributions however differ markedly between the two models. For Model 1f, results indicate a substantial increase in the difference of accumulation rates after the change of motion. This rate asymmetry suggests that the accumulation process is not veridical since a 
symmetric change of stimulus leads to a non-symmetric change in the underlying accumulation process. In Model 2f, we see a substantial compression of the two drift rates after the switch so that drift rates for the two alternatives are nearly the same. This however is an artefact of removing the rate delay, or rather setting it to 0 .

This compression of drift rates in the absence of a rate delay is consistent with data in Figure 2c. That data indicates there is a time delay between presentation and incorporation of the new information. By removing the rate delay, the model is being forced to estimate the post switch drift rates based on an average of early and late post change responses. Because early post change responses are most likely based on the initial pre-change stimulus information, they are essentially washing each other out. Consider a left-then-right motion switch trial with a switch occurring at time $t_{0}$ where an intrinsic rate delay of $t_{\text {rate }}$ is present. If we force the rate delay to be 0 in the model, then the post change drift rates must accommodate choices made during the intrinsic rate delay $t_{\text {rate }}$ (which will favor the pre-change stimulus direction) and choices made after the intrinsic delay (which will favor the post-change stimulus direction). Since each motion direction is preferred for some period of time, the post switch drift rates appear to be nearly the same. This strongly suggests that not only is the change of drift rates vital in accounting for the data, but so is a sufficiently long rate delay.

The post switch threshold and threshold delay estimation in Figure 3b also provide insights. For both model variants, there is more uncertainty in threshold than rate estimates, but for Model 2's changed-threshold estimate $\left(\mu_{b 2}\right)$ this uncertainty becomes extreme, with a close to flat distribution between 2.5 and 4 . Further examination of the threshold delay $\left(\mu_{\text {thresh }}\right)$ reveals estimates that crowd at the upper bound of the prior $(3 \mathrm{~s})$ and which are always greater than $2.5 \mathrm{~s}$. Recalling that all trials are terminated after $2 \mathrm{~s}$, this indicates the model is fitting both the threshold delay and the post switch threshold parameters as nuisance parameters that do not impact the fit. The threshold delay 
$\left(t_{\text {thresh }}\right)$ is so long that the threshold cannot change before the trial terminates. As a result, the data cannot impact estimation of $\mu_{b 2}$, resulting in a more uniformly distributed posterior. This suggests that a change of threshold does not provide a good accounting of the data.

In contrast to Model 2f's $\mu_{\text {thresh }}$ parameter, estimates of Model 1f's $\mu_{\text {rate }}$ parameter is well away from the prior bounds and relatively peaked (note the very different scales for the two delay panels in Figure 3). On average the estimated delay in the integration of new information is greater than $0.4 \mathrm{~s}$. This delay is the source of the lag effect evident in Figure 2c). Furthermore, the posterior hyper standard deviation $\sigma_{\text {rate }}$ had a mean value of only $0.076 \mathrm{~s}$, indicating that there is a relatively low level of participant variability in the rate delay.

Interestingly, when a delayed rate change is combined with an immediate threshold change where $t_{\text {thresh }}=0$ (Model 3f, parameter estimates not shown), the rate differential agrees most closely with the purely delayed rate model (1.2 before and 2 after). Further, the distributions of $\mu_{b 1}$ and $\mu_{b 2}$ are estimated as almost identical, with a very small increase in the mean of 2.5 for $\mu_{b 1}$ and a mean of 2.6 for $\mu_{b 2}$. Thus, when a threshold increase was forced to occur, the magnitude of that increase was estimated as relatively negligible.

In summary, when a change of threshold is allowed to occur, the data forces the delay in that change to be so long that it occurs after the trial terminates so that in effect, it never occurs. When the change of threshold is forced to occur, the data constrains the new threshold to be nearly identical to the threshold prior to the change of motion. Taken together, these results support selection of Model 1f as best reflecting the data. Further analyses focus on this model; we first consider the quality of its fit to data, and then examine individual differences in its parameter estimates, and their relationship to individual differences in performance. 
The Piecewise Linear Ballistic Accumulator 35

\section{Quality of fit}

Figure 4 provides a visual assessment of the quality of the fit by Model 1f. For each participant, we computed the posterior mean value for the individual level parameters and examined the quality of fit for each participant. In panel $a$, we aggregate data and model predictions for all participants over blocks 4-18. Each black (respectively grey) data point represents the predicted and actual probability of choosing the pre-switch correct choice (C1) (respectively the post-switch correct choice, C2) for an individual block and participant, so there are $31 \times 15$ data points for each color (31 participants and 15 blocks). Note the actual probabilities for each block are computed over only 72 trials, so a significant degree of randomness is expected for each observation. Nonetheless, we see a clear correlation $(r=0.63)$ between predicted and actual choice probabilities (no $p$-value reported due to the large number of data points rendering it moot).

Figures 4 b-d show actual (histograms) and predicted (lines) response time distributions for three separate participants. This data is again aggregated over blocks 4-18 with black (respectively grey) indicating response times for the pre-switch correct choice $(\mathrm{C} 1)$ (respectively the post-switch correct choice, C2). In order to make a direct comparison between model predictions and data, for each participant we numerically recreated the experiment with the posterior mean parameters. That is, 15 blocks were simulated using the precise recorded switch times for each participant (with 1000 instead of 72 trials to reduce randomness). Simulated response time data was then similarly aggregated over the 15 blocks and scaled appropriately for plotting. These results show a diversity of response time distributions, each of which is well accounted for by this model. In particular, panel $c$ shows a bimodal response distribution for choice $\mathrm{C} 2$ where the first peak corresponds to incorrect responses prior to the switch and the second peak to correct responses after the switch. Although we only present data for these three participants, we visually assessed the fit for all participants and found similar agreement for 30 of the 31 . 
The remaining participant exhibited nearly uniform posterior distributions for post switch, individual level parameters, indicating poor fit.

Figures $4 \mathrm{e}$ and $4 \mathrm{f}$ show a comparison of quartile accuracy between the data and model predictions for stationary and switch trials respectively. For 4e, quartile accuracy was computed (for each participant individually) from the full RT distribution for all stationary trials, irrespective of the experimental block. Box plots show the distribution of accuracy over participants. For 4f, quartiles were computed from the RT distribution containing switch trials where responses occur after the change of information (similar to Figure 2c). To generate model predictions, the mean of the posterior distribution was computed for each participant. These parameters were then used to run a synthetic version of the experiment and generate a synthetic data set. Quartile accuracy for both stationary and switch trials was then computed for these synthetic data and plotted along side participant accuracy data. Results indicate that the model accounts well for the general trends in the data. There is a small uptick in accuracy on stationary trials from q1 to q2 that the model does not capture. It does however capture the trend toward reduced accuracy from q2-q4 on stationary trials and the substantial increase in accuracy from q3-q4 on switch trials.

We next asked how various parameters correlate with one other. At the participant level, there are a number of strong correlations. This is illustrated in Figure 5 for one participant. Such strong correlations are common in evidence accumulation models, including the LBA (Turner et al., 2013), since their dynamics are only partially constrained by behavioural data (e.g., accumulation trajectories are only observed through their effect at their end point). This is a common modelling problem in many contexts where sparse or insufficient observations are used to estimate parameters of complex models. Due to the inability to precisely determine parameters of these models (either using Bayesian or frequentists methods), they are often referred to as "sloppy models" 
(Gutenkunst et al., 2007; Apgar, Witmer, White, \& Tidor, 2010).

Despite these correlations, the post switch drift rates appear to be virtually uncorrelated with all other system parameters, indicating they are specific in the sense that their values correspond to post switch rates of accumulation and are not strongly influenced by other factors. This is not surprising given the structure of the model and the structure of the data used to parameterise it. Perhaps more surprisingly, results (not presented) indicate there is very little correlation between hyper mean parameters; the only correlation above 0.2 was between $\mu_{A}$ and $\mu_{b}$. This indicates there are no structural correlations at the population level.

\section{Individual Differences}

In order to examine the relationship between individual differences in performance on the task and rate asymmetry, we defined a measure of the difference in average rate differentials before and after the rate change for each participant:

$$
D^{p}=\left(\bar{\mu}_{w 2}^{p}-\bar{\mu}_{w 1}^{p}\right)-\left(\bar{\mu}_{v 1}^{p}-\bar{\mu}_{v 2}^{p}\right)
$$

where individual level parameters are used in this definition. A positive value of this asymmetry measure indicates that after the rate change there is an increased differential rate (i.e., an increased ability to discriminate the motion direction). As shown in the first row of Table 2, we found a strong positive correlation between the asymmetry measure and accuracy on the stationary trials of the direction determination task. That is, participants whose performance in the stationary case was superior displayed a greater asymmetry, in the form of an increase in their ability to discriminate the changed direction of motion.

Interestingly, however, the asymmetry measure was not significantly correlated with the ability to detect the stimulus change in block 20, as shown in the second row of Table 2. We also correlated each participant's posterior mean for the rate delay with 
performance on the switch detection task and found no relation (second row of Table 2), which reflects the relatively low level of subject-level variability in this delay.

Further, we correlated the asymmetry measure and the posterior mean of the rate delay with mean RTs on stationary and switch trials of the direction determination task. For the mean post switch RT, only the trials where responses occur after the change of motion were included. For those trials, rather than considering the RT itself, the total elapsed time after the switch (e.g. RT - switch time) is used. As shown in Table 2, mean RTs for both stationary and switch trials were clearly correlated with the asymmetry measure (left column), but not the rate delay (right column). In sum, the rate asymmetry is strongly related to performance measures (response times and accuracy), while the rate delay appears to be independent of these measures.

Figure 6 plots the estimated distribution of individual differences in the rate delay and asymmetry measures. The histograms take into account both variability among participants and uncertainty about the value of the measures for each participant. There is clear evidence for a strong rate delay that is quite consistent over participants. In contrast there is much stronger individual variation in the asymmetry. The majority of participants are predicted to have a positive asymmetry, with some evidence of a separate mode for participants with very high asymmetry. However, it is also possible that a minority of participants will display no asymmetry or even a slightly negative asymmetry, as $16 \%$ of the mass of the histogram on the right of Figure 6 is less than zero.

\section{General Discussion}

In the course of our day to day lives, we encounter numerous situations that require decisions based on sequentially encountered pieces of conflicting information. Our goal in the present work was to develop a new quantitative framework appropriate for modelling such situations, the Piecewise Linear Ballistic Accumulator (PLBA), and to apply it to a 
particular situation in order to understand how participants adapt to new information. The particular situation we chose to use involved a random dot motion (RDM) task, where the direction of dot motion changed once within a trial, from left to right or visa versa. Our broader aim was to show that the PLBA model provides psychological insights into decision making with non-stationary stimuli that are not otherwise available, and hence to encourage its wider application.

We built the PLBA model by elaborating Brown and Heathcote's (2008) LBA model of decisions based on stationary stimuli. The LBA has been shown to accommodate a range of benchmark binary choice phenomena, including predicting fast and slow errors and the shape of speed-accuracy tradeoff curves (Brown \& Heathcote, 2008). It has also been successful at accounting for neural data (Forstmann et al., 2008, 2010) and data from complex decision paradigms involving more than one response (Hawkins et al., 2013), decisions with more than two choices (e.g., Brown \& Heathcote, 2008; van Maanen et al., 2012) including choices based on multi-attribute stimuli (Trueblood, Brown, \& Heathcote, 2014). The LBA's mathematical and computational tractability has underpinned its widespread application, and it is these characteristics that made it practical to elaborate in order to address situations where decisions are based on non-stationary stimuli. A second essential ingredient was recent developments in Probability Density Approximation (PDA) based Approximate Bayesian Computation (Turner \& Sederberg, 2014; Holmes, 2015) and methods for sampling from models with correlated posteriors (Turner et al., 2013).

In order to understand how the decision process is updated after a change of information, we constructed a series of hierarchal PLBA models of increasing complexity, each of which makes a different assumption on the decision update process, and used Bayesian methods to fit each of these models to data from the RDM direction-determination task. The models allowed examination of five potential mechanisms (and combinations thereof) that could be at work when decisions are made 
about changing information : immediate or delayed changes in the rate of evidence accumulation, immediate or delayed changes in evidence thresholds, and carryover effects causing a non-veridical representation of the changed information. Analysis based on model selection and examination of posterior parameter estimates supported a model with two of these features.

First, there was a surprisingly substantial delay, of the order of $0.45 \mathrm{~s}$ before the change of stimulus direction affected the rate of evidence accumulation. The presence of a large delay explained raw choice proportion results that the new information only affected choices in the longest quartile of responses occurring after the stimulus switched. A second finding - one that is perhaps even more surprising and certainly not something that could be inferred based on the raw data alone - was that ability to discriminate motion direction improved after the changed information reached the decision process. The ability of the PLBA model to make a correct choice depends on how much greater the rate is for the accumulator that matches the stimulus than for the alternative accumulator. Our stimuli were symmetric (i.e., motion is equal in magnitude but opposite in direction before and after the change), so that if the rates are veridical, then discrimination should be symmetric. However we found that when the rates eventually change, the difference in rates between the matching and mismatching accumulators is much larger than before the change. It is possible is that the larger rate after the change is an attempt to compensate for the delay, but there was relatively little variation in the delay or correlation between the delay and the increased rate over participants $(r=0.011, p=0.95)$, so any potential compensatory mechanism is not sensitive to individual differences.

It is possible that the rate delay and improved discrimination could be the spurious result of fitting the (misspecified) PLBA model to a DDM data generating process similar to those proposed by White et al. (2011) and Hübner et al. (2010). To examine this possibility we simulated data from a DDM where the drift rate switched from negative to 
positive (but with equal absolute magnitude) half way through the decision. When we fit the PLBA to this simulated data, there was only a negligible rate delay (less than $10 \%$ of the mean decision time), and slightly reduced (rather than improved) discrimination after the switch. Although we did not investigate all possible combinations of DDM parameters ${ }^{4}$ it seems unlikely that this sort of model misspecification could explain either the substantial delay, or the asymmetry, that we inferred from fits of the PLBA to our data.

When thought of in terms of the primacy and recency constructs that have framed conceptualisations of non-stationary decision making in the past (e.g., Usher \& McClelland, 2001; Brown \& Heathcote, 2005b), the delay and asymmetry findings appear contradictory. Delay is most consistent with a competitive mechanism, whereby accumulated evidence in the decision process suppresses new contradictory information, so it takes an extended period of time before that information has an impact (Tsetsos et al., 2012). However, the direction of the rate asymmetry is more consistent with a recency effect where later arriving information has a stronger impact. One possible resolution is offered by a middle level of competition; Tsetsos et al. showed that in a region between linear accumulation and very strong competition the rich and flexible dynamics of LCA can exhibit a change from a primacy to recency pattern over the transition from faster to slower response-time trials (see their Figure 5).

Our aim here is not to resolve such issues, but rather to demonstrate the useful and revealing nature of a PLBA based analysis. However, it is tempting to speculate, and useful to do so in order to underline the inherit limitations in our approach, or indeed any approach that models only the decision process. As we previously noted, rate effects can have two sources, from the dynamics of the processes that encode stimuli and from the dynamics of the decision process itself. Using this distinction, one potential resolution to our findings is that the delay results from competition in the decision process, whereas the 
rate asymmetry emerges from the dynamics of stimulus encoding.

The latter speculation is based on the finding that the increased rate difference in the changed rates (see Figure 3) was mainly due to a marked depression in the rate associated with the mismatching accumulator (on average from 2.1 before the change to 1.3 after the change), with only a small increase in the rate for the matching accumulator (on average 3.4 before the change to 3.7 after the change). This depression might be due to a depletion of neurotransmitters in cells tuned to represent the initial motion direction (Carpenter \& Grossberg, 1990), so that their activity in response to the changed motion is depressed relative to the activity of cells tuned to the new motion direction. Such suppression is consistent with the motion aftereffect, where prolonged adaptation to motion in one direction leads to the appearance of motion in the opposite direction (Anstis et al., 1998).

A depletion mechanism might also be included in an elaboration of the dynamics of nonlinear decision models such as those of Usher and McClelland (2001) or Brown and Heathcote (2005b). Similarly, a delay might emerge from competitive dynamics between cell populations tuned to different motion directions. Further experimentation - for example exploring whether the effects we observed occur with other types of stimuli, perhaps randomly mixing different stimulus types so that an invariant decision process can be assumed - will be required to disentangle these possibilities. The point we wish to emphasise here is that PLBA modelling provides a method of asking such questions and can contribute to answering them, thus providing a deeper understanding of how decisions are made about non-stationary stimuli. That is, we advocate the use of the PLBA in the same spirit as the LBA, tractable approximation that can be applied to model non-stationary processing - much as Euler methods can provide a useful way to model non-linear dynamics.

The stimuli which we used in our experiment produced a change that could be 
discriminated by participants, but this did not seem to lead to any reactivity to the change in the form of an adjustment in the parameters of their decision processes, in particular the evidence threshold. However, the change was not particularly easy to discriminate, so it is possible that reactivity may occur with more noticeable changes, or perhaps with decisions made over longer time scales where there is more time to adjust thresholds. The general form of the PLBA model illustrated in Figure 1 can be used to explore such possibilities.

A potential criticism of the PLBA model is that it is very flexible. Figure 4 demonstrates this flexibility by showing the model's ability to fit negatively skewed and bimodal RT distributions. Jones and Dzhafarov (2014) recently raised the issue of flexibility with respect to LBA and DDM models that do not make definite assumptions about the form of variability in their parameters from trial to trial. Heathcote, Wagenmakers, and Brown (2014) showed that when the conventional distributional assumptions are made for the LBA that it is not able to fit negatively skewed distributions and the same is true for bimodal distributions unless a mixture of processes is present (e.g.,Cassey, Heathcote, \& Brown, 2014). Given the greater flexibility of the PLBA, we recommend it be assessed with model-selection methods that can provide appropriate penalties for over-fitting. Our use of DIC here goes some way to fulfilling this criterion, although it is less than ideal given it is known to asymptotically prefer overly complex models. However, even if a better criterion, such as Bayes Factors, were used, we think converging evidence from considerations like the plausibility and coherence of posterior parameter estimates are also desirable.

\section{Conclusions}

In sum, the PLBA can be used to explore process-level hypotheses for how non-stationary information influences the decision process. Using eight variants of the 
PLBA, we tested hypotheses about how drifts and thresholds might change in response to external changes of information. Our results revealed that changes in drift rates rather than changes in threshold provide the best explanation for how people adapt and integrate new perceptual information. Future research could explore the generality of these findings in both perceptual and high-level decision-making. We believe that the PLBA in combination with efficient approximate Bayesian methods provide an excellent way to address such questions. 
The Piecewise Linear Ballistic Accumulator 45

\section{References}

Anstis, S., Verstraten, F. A., \& Mather, G. (1998). The motion aftereffect. Trends in cognitive sciences, 2(3), 111-117.

Apgar, J. F., Witmer, D. K., White, F. M., \& Tidor, B. (2010). Sloppy models, parameter uncertainty, and the role of experimental design. Molecular BioSystems, 6(10), 1890-1900.

Atkinson, K. A. (1989). An introduction to numerical analysis (2nd ed.). John Wiley \& Sons.

Ball, K., \& Sekuler, R. (1982). A specific and enduring improvement in visual motion discrimination. Science, 218(4573), 697-698.

Botvinick, M. M., Braver, T. S., Barch, D. M., Carter, C. S., \& Cohen, J. D. (2001). Conflict monitoring and cognitive control. Psychological Review, 108, 624-652.

Britten, K. H., Shadlen, M. N., Newsome, W. T., \& Movshon, J. A. (1992). The analysis of visual motion: a comparison of neuronal and psychophysical performance. The Journal of Neuroscience, 12(12), 4745-4765.

Britten, K. H., Shadlen, M. N., Newsome, W. T., \& Movshon, J. A. (1993). Responses of neurons in macaque mt to stochastic motion signals. Visual neuroscience, 10(06), $1157-1169$.

Brown, S. D., \& Heathcote, A. (2005a). A ballistic model of choice response time. Psychological review, 112(1), 117.

Brown, S. D., \& Heathcote, A. (2005b). Practice Increases the Efficiency of Evidence Accumulation in Perceptual Choice. Journal of Experimental Psychology: Human Perception and Performance, 31(2), 289-298.

Brown, S. D., \& Heathcote, A. (2008). The simplest complete model of choice response time: Linear ballistic accumulation. Cognitive psychology, 57(3), 153-178.

Brown, S. D., Ratcliff, R., \& Smith, P. L. (2006, August). Evaluating methods for 
The Piecewise Linear Ballistic Accumulator 46

approximating stochastic differential equations. Journal of Mathematical Psychology, 50(4), 402-410.

Brunton, B. W., Botvinick, M. M., \& Brody, C. D. (2013, April). Rats and Humans Can Optimally Accumulate Evidence for Decision-Making. Science, 340(6128), 95-98.

Busemeyer, J. R., \& Diederich, A. (2002). Survey of decision field theory. Mathematical Social Sciences, 43(3), 345-370.

Busemeyer, J. R., \& Townsend, J. T. (1993). Decision field theory: a dynamic-cognitive approach to decision making in an uncertain environment. Psychological review, $100(3), 432-459$.

Carpenter, G. A., \& Grossberg, S. (1990). ART 3: Hierarchical search using chemical transmitters in self-organizing pattern recognition architectures. Neural Networks, 3(2), 129-152.

Cassey, P., Heathcote, A., \& Brown, S. D. (2014, July). Brain and Behavior in Decision-Making. PLoS Computational Biology, 10(7), e1003700.

Cisek, P., Puskas, G. A., \& El-Murr, S. (2009). Decisions in changing conditions: The urgency-gating model. The Journal of Neuroscience, 29(37), 11560-11571.

Diederich, A. (1997). Dynamic stochastic models for decision making under time constraints. Journal of Mathematical Psychology, 41(3), 260-274.

Diederich, A. (2008). A further test of sequential-sampling models that account for payoff effects on response bias in perceptual decision tasks. Perception $\mathscr{G}$ Psychophysics, $70(2), 229-256$.

Diederich, A., \& Busemeyer, J. R. (1999). Conflict and the stochastic-dominance principle of decision making. Psychological Science, 10(4), 353-359.

Diederich, A., \& Busemeyer, J. R. (2006). Modeling the effects of payoff on response bias in a perceptual discrimination task: Bound-change, drift-rate-change, or two-stage-processing hypothesis. Perception 85 Psychophysics, 68(2), 194-207. 
The Piecewise Linear Ballistic Accumulator 47

Donkin, C., Brown, S. D., \& Heathcote, A. (2009). The overconstraint of response time models: Rethinking the scaling problem. Psychonomic bulletin $\&$ review, 16(6), $1129-1135$.

Edwards, W. (1965). Optimal strategies for seeking information: Models for statistics, choice reaction times, and human information processing. Journal of Mathematical Psychology, 2, 312-329.

Epanechnikov, V. (1969). Non-parametric estimation of a multivariate probability density. Theory of Probability and Its Applications, 14(1), 153-158. Retrieved from http://epubs.siam.org/doi/abs/10.1137/1114019 doi: 10.1137/1114019

Erikesn, C. W., \& Eriksen, C. W. (1974). Effects of noise letters upon the identification of a target letter in a nonsearch task. Perception $\&$ Psychophysics, 16, 143-149.

Forstmann, B. U., Anwander, A., Schäfer, A., Neumann, J., Brown, S. D., Wagenmakers, E.-J., .. Turner, R. (2010). Cortico-striatal connections predict control over speed and accuracy in perceptual decision making. Proceedings of the National Academy of Sciences, 107(36), 15916-15920.

Forstmann, B. U., Dutilh, G., Brown, S. D., Neumann, J., von Cramon, D. Y., Ridderinkhof, K. R., \& Wagenmakers, E. J. (2008). Striatum and pre-sma facilitate decision-making under time pressure. Proceedings of the National Academy of Science, $105,17538-17542$

Gold, J. I., \& Shadlen, M. N. (2001). Neural computations that underlie decisions about sensory stimuli. Trends in Cognitive Sciences, 5(1), 10-16.

Gold, J. I., \& Shadlen, M. N. (2007). The neural basis of decision making. Annu. Rev. Neurosci., 30, 535-574.

Gutenkunst, R. N., Waterfall, J. J., Casey, F. P., Brown, K. S., Myers, C. R., \& Sethna, J. P. (2007). Universally sloppy parameter sensitivities in systems biology models. PLoS computational biology, 3(10), e189. 
The Piecewise Linear Ballistic Accumulator 48

Hawkins, G. E., Marley, A. A. J., Heathcote, A., Flynn, T. N., Louviere, J. J., \& Brown, S. D. (2013, October). Integrating Cognitive Process and Descriptive Models of Attitudes and Preferences. Cognitive Science, 38(4), 701-735.

Heath, R. A. (1981). A tandem random walk model for psychological discrimination. British Journal of Mathematical and Statistical Psychology, 34(1), 76-92. Retrieved from http://dx.doi.org/10.1111/j.2044-8317.1981.tb00619.x doi: 10.1111/j.2044-8317.1981.tb00619.x

Heathcote, A., Wagenmakers, E. J., \& Brown, S. D. (2014). The falsifiability of actual decision-making models. Psychological Reivew, 121, 676-678.

Hiris, E., \& Blake, R. (1992). Another perspective on the visual motion aftereffect. Proceedings of the National Academy of Sciences, 89(19), 9025-9028.

Hogarth, R. M., \& Einhorn, H. J. (1992). Order effects in belief updating: The belief-adjustment model. Cognitive psychology, 24(1), 1-55.

Holmes, W. R. (2015). A practical guide to the probability density approximation (pda) with improved implementation and error characterization. Journal of Mathematical Psychology, 68-69, 13-24.

Hübner, R., Steinhauser, M., \& Lehle, C. (2010). A dual-stage two-phase model of selective attention. Psychological Review, 117, 759-784.

Huk, A. C., \& Shadlen, M. N. (2005). Neural activity in macaque parietal cortex reflects temporal integration of visual motion signals during perceptual decision making. The Journal of neuroscience, 25(45), 10420-10436.

Insabato, A., Dempere-Marco, L., Pannunzi, M., Deco, G., \& Romo, R. (2014). The influence of spatiotemporal structure of noisy stimuli in decision making. $P L o S$ computational biology, 10(4), e1003492.

Jones, M., \& Dzhafarov, E. N. (2014). Unfalsifiability and mutual translatability of major modeling schemes for choice reaction time. Psychological review, 121(1), 1. 
Jong, R. D., Liang, C. C., \& Lauber, E. (1994). Conditional and unconditional automaticity: a dual-process model of effects of spatial stimulus-response correspondence. Journal of Experimental Psychology: Human Perception and Performance, 20, 731-750.

Kiani, R., Hanks, T. D., \& Shadlen, M. N. (2008). Bounded integration in parietal cortex underlies decisions even when viewing duration is dictated by the environment. The Journal of Neuroscience, 28(12), 3017-3029.

Krajbich, I., Armel, C., \& Rangel, A. (2010). Visual fixations and the computation and comparison of value in simple choice. Nature neuroscience, 13(10), 1292-1298.

Krajbich, I., \& Rangel, A. (2011). Multialternative drift-diffusion model predicts the relationship between visual fixations and choice in value-based decisions. Proceedings of the National Academy of Sciences, 108(33), 13852-13857.

Luce, R. D. (1986). Response times: Their role in inferring elementary mental organization3. New York, NY: Oxford University Press.

McKenzie, C. R. M., Lee, S. M., \& Chen, K. K. (2002). When negative evidence increases confidence: Change in belief after hearing two sides of a dispute. Journal of Behavioral Decision Making, 15, 1-18.

Ratcliff, R. (1978). A theory of memory retrieval. Psychological Review, 85, 59-108.

Ratcliff, R. (1980). A note on modeling accumulation of information when the rate of accumulation changes over time. Journal of Mathematical Psychology, 21(2), $178-184$

Ratcliff, R., \& McKoon, G. (2008). The diffusion decision model: Theory and data for two-choice decision tasks. Neural Computation, 20(4), 873-922.

Ratcliff, R., \& Rouder, J. N. (1998). Modeling response times for two-choice decisions. Psychological Science, 9(5), 347-356.

Ridderinkhof, R. (2002). Micro- and macro-adjustments of task set: activation and 
The Piecewise Linear Ballistic Accumulator 50

suppression in conflict tasks. Psychological Research, 66, 312-323.

Shadlen, M. N., \& Newsome, W. T. (1996). Motion perception: Seeing and deciding. Proceedings of the National Academy of Sciences, 93(2), 628-633.

Silverman, B. W. (1982). Algorithm as 176: Kernel density estimation using the fast fourier transform. Journal of the Royal Statistical Society. Series C (Applied Statistics), 31(1), pp. 93-99.

Silverman, B. W. (1986). Density estimation for statistics and data analysis (Vol. 26). CRC press.

Simon, J. R., \& Rundell, A. P. (1967). Auditory s-r compatibility: The effect of an irrelevant cue on information processing. Journal of Applied Psychology, 51, $300-304$.

Smith, P. L., \& Ratcliff, R. (2004). Psychology and neurobiology of simple decisions. Trends in neurosciences, $27(3), 161-168$.

Spiegelhalter, D. J., Best, N. G., Carlin, B. P., \& Van Der Linde, A. (2002). Bayesian measures of model complexity and fit. Journal of the Royal Statistical Society: Series B (Statistical Methodology), 64(4), 583-639.

Storn, R., \& Price, K. (1997). Differential evolution-a simple and efficient heuristic for global optimization over continuous spaces. Journal of global optimization, 11(4), $341-359$

Ter Braak, C. J. (2006). A markov chain monte carlo version of the genetic algorithm differential evolution: easy bayesian computing for real parameter spaces. Statistics and Computing, 16(3), 239-249.

Trueblood, J. S., Brown, S. D., \& Heathcote, A. (2014). The multi-attribute linear ballistic accumulator model of context effects in multi-alternative choice. Psychological Review, 121, 179-205.

Tsetsos, K., Gao, J., McClelland, J. L., \& Usher, M. (2012). Using time-varying evidence 
to test models of decision dynamics: Bounded diffusion vs. the leaky competing accumulator model. Frontiers in Neuroscience, 6 .

Tsetsos, K., Usher, M., \& McClelland, J. L. (2011). Testing multi-alternative decision models with non-stationary evidence. Frontiers in neuroscience, 5.

Turner, B. M., \& Sederberg, P. B. (2014). A generalized, likelihood-free method for posterior estimation. Psychonomic bulletin \& review, 21, 227-250.

Turner, B. M., Sederberg, P. B., Brown, S. D., \& Steyvers, M. (2013). A method for efficiently sampling from distributions with correlated dimensions. Psychological methods, 18(3), 368-384.

Usher, M., \& McClelland, J. L. (2001). The time course of perceptual choice: the leaky, competing accumulator model. Psychological Review, $108(3)$, 550-592.

van Maanen, L., Grasman, R. P., Forstmann, B. U., Keuken, M. C., Brown, S. D., \& Wagenmakers, E.-J. (2012). Similarity and number of alternatives in the random-dot motion paradigm. Attention, Perception, \& Psychophysics, 74(4), 739-753.

White, C. N., Ratcliff, R., \& Starns, J. J. (2011). Diffusion models of the flanker task: Discrete versus gradual attentional selection. Cognitive Psychology, 63(4), 210-238.

Winkel, J., Keuken, M. C., Van Maanen, L., Wagenmakers, E.-J., \& Forstmann, B. U. (2014). Early evidence affects later decisions: Why evidence accumulation is required to explain response time data. Psychon Bull Rev, 21, 777-784. 
The Piecewise Linear Ballistic Accumulator 52

\section{Appendix A}

\section{PLBA Pseudo-analytic Likelihood}

While we have not used it here for computational reasons, it is possible to compute a pseudo analytic likelihood function for the PLBA model with a fixed threshold (Model 1f). The basic assumption of Model $1 \mathrm{f}$ is that after a change of information, evidence accumulation rates adjust. Mathematically, this is modelled by assuming that post switch drift rates are drawn from new normal distributions. In order to compute this density function, we proceed in much the same way as in the computation of the standard LBA density function (Brown \& Heathcote, 2005a). Let $F_{i}(T)$ be the cumulative density function $(\mathrm{CDF})$ for the time at which an individual accumulator $i$ terminates at the fixed threshold. If the change of accumulation rate occurs at time $t_{0}$, then the state of the accumulator is

$$
x_{i}(T)= \begin{cases}x_{0}+v_{i} T, & T<t_{0} \\ x_{0}+v_{i} t_{0}+w_{i}\left(T-t_{0}\right), & T>t_{0},\end{cases}
$$

where $v_{i}, w_{i}$ are the pre and post switch drift rates for that accumulator, each drawn from an associated normal distribution.

At this point it is useful to break the time domain into two pieces, $T<t_{0}$ and $T>t_{0}$. If $T<t_{0}$, then the second accumulation rate never gets used and the quantity $F_{i}(T)$ is determined by the standard LBA formula

$$
F_{i}(T)=K_{1}\left(T \mid A, b, \mu_{v}, \sigma\right)
$$


where

$$
\begin{aligned}
K_{1}(t \mid A, B, \mu, \sigma)=1 & +\frac{B-A-\mu t}{A} \Phi\left(\frac{B-A-\mu t}{\sigma t}\right)+\frac{\mu t-B}{A} \Phi\left(\frac{B-\mu t}{\sigma t}\right) \\
& +\frac{\sigma t}{A} \phi\left(\frac{B-A-\mu t}{\sigma t}\right)-\frac{\sigma t}{A} \phi\left(\frac{B-\mu t}{\sigma t}\right),
\end{aligned}
$$

is the usual LBA kernel. Here, it is assumed that $v_{i} \sim N\left(\mu_{v i}, \sigma\right)$ and that $x_{0} \sim U(0, A)$.

For $T>t_{0}$, things are more complicated. In this case

$$
\begin{aligned}
F_{i}(T) & =\int_{0}^{\infty} P\left(x_{i}(T)>b \mid v_{i}\right) \phi\left(v_{i} \mid \mu_{v i}, \sigma\right) d v_{i} \\
& =\int_{0}^{(b-A) / t_{0}} P_{1} \phi\left(v_{i} \mid \mu_{v i}, \sigma\right) d v_{i}+\int_{(b-A) / t_{0}}^{b / t_{0}} P_{2} \phi\left(v_{i} \mid \mu_{v i}, \sigma\right) d v_{i}+\int_{b / t_{0}}^{\infty} P_{3} \phi\left(v_{i} \mid \mu_{v i}, \sigma\right) d v_{i} .
\end{aligned}
$$

Here $\phi$ is the PDF for the normal distribution. This integral has been broken into three pieces since the quantity $P$ has different forms denoted by $P_{1,2,3}$ in these different regions. After some technical calculation that follows Brown and Heathcote (2005a), it is direct to show that

$$
\begin{aligned}
& P_{1}=K_{1}\left(T-t_{0} \mid A, b-v_{i} t_{0}, \mu_{w i}, \sigma\right), \\
& P_{2}=K_{2}\left(T-t_{0} \mid A, b-v_{i} t_{0}, \mu_{w i}, \sigma\right), \\
& P_{3}=1,
\end{aligned}
$$

where $K_{1}$ is the standard LBA kernel and $K_{2}$ is a variant of it

$$
\begin{aligned}
K_{2}(t \mid A, B, \mu, \sigma)=1 & +\frac{B-A-\mu t}{A} \Phi\left(\frac{-\mu}{\sigma}\right)+\frac{\mu t-B}{A} \Phi\left(\frac{B-\mu t}{\sigma t}\right) \\
& +\frac{\sigma t}{A} \phi\left(\frac{-\mu}{\sigma}\right)-\frac{\sigma t}{A} \phi\left(\frac{B-\mu t}{\sigma t}\right) .
\end{aligned}
$$

Note that we are referring to these as "kernels" as they are acting as integration kernels in 
this description of $F_{i}(T)$. Additionally, it is of note that the third integral in this expression can be simplified so that

$$
\int_{b / t_{0}}^{\infty} P_{3} \phi\left(v \mid \mu^{v}, \sigma\right)=1-\Phi\left(\frac{b}{t_{0}}, \mu^{v}, \sigma\right)=1-\Phi\left(\frac{b-\mu^{v} t_{0}}{\sigma t_{0}}\right) .
$$

In this form, the first integral is very similar to a convolution of the LBA kernel $\left(K_{1}\right)$ against the normal distribution. The second integral has a similar form but with a slightly different kernel. In this expression, the functions $K_{1,2}$ are well defined and the necessary integrals in Equ. (12) can be readily computed with standard integral routines. Once expressions for the PLBA CDF's are obtained (e.g. $F_{1}, F_{2}$ ), the associated PDF's can be computed as $f_{i}=F_{i}^{\prime}$. Subsequently, the defective distributions describing the choice probabilities can be computed as

$$
g_{i}\left(T_{k}\right):=f_{i}\left(T_{k}\right) \prod_{j \neq i}\left(1-F_{j}\left(T_{k}\right)\right) .
$$

The caveat to using this solution is that it requires numerically computing the integrals in Equ. (12). While this is not technically difficult, our investigations indicate that evaluating the density function in this way is orders of magnitude slower than using the probability density approximation used in this paper, making it impractical to use in a Bayesian parameter estimation context. Numerical computation of derivatives is also required when evaluating $f_{i}$, however this appears to be less problematic than integral evaluation. 
The Piecewise Linear Ballistic Accumulator 55

\section{Appendix B}

\section{Estimation Methods}

\section{Hierarchal Bayesian Parameter Estimation}

For each model, we make the assumption that trials are identical and independent so that for each participant

$$
L\left(R T, R C \mid \theta^{p}\right)=\prod_{j=1}^{N} L\left(T_{j}^{p}, C_{j}^{p} \mid \theta^{p}\right),
$$

where $T_{j}^{p}, C_{j}^{p}$ are the response time and choice for trial $j$ and participant $p$. Experimental blocks 1-3 and 19-20 serve alternative purposes and so only data from blocks 4-18 are used for the fitting process. The data for each participant is thus comprised of $N=15 \times 72$ response time and choice doublets.

For Model 0, where no switch is included, this likelihood can be analytically evaluated (Brown \& Heathcote, 2008) since the drift rates do not change. All model variants treat stationary trials identically to the LBA and so the likelihood of those trials can also be analytically evaluated. However, while the PLBA does emit an analytic representation for the likelihood $L\left(T_{j}^{p}, C_{j}^{p} \mid \theta^{p}\right)$, it is computationally problematic to compute. We instead use a more computationally efficient variant of a recently developed non-parametric approximate Bayesian computation method (Turner \& Sederberg, 2014) to estimate the likelihood of switch trials and fit the full hierarchal model.

Likelihood approximation. Consider Model 1f where the rate switch occurs at fixed time $t_{0}$. The goal is to compute $L\left(T-t_{e r}, C \mid b, A, \mu_{v 1}, \mu_{v 2}, \mu_{w 1}, \mu_{w 2}, t_{0}+t_{\text {rate }}\right)$ where $T-t_{e r}$ is the decision time, $C$ the choice, and $t_{0}+t_{\text {rate }}$ is the time at which the participant processes the switch. For simplicity and brevity of notation, sub and super scripts have been omitted. The basic procedure for approximating this likelihood is to 1) 
draw a large number of samples $\left(N_{s}\right)$ from the joint choice and response time distribution and 2) use kernel density estimation (Silverman, 1986) to approximate the likelihood from these samples.

To draw the samples for a fixed set of parameters, it is sufficient to run $N_{s}$ pairs of accumulators, and for each set, record the choice and response time. This will produce a set of samples denoted $\left\{\tau_{k}, c_{k}\right\}_{k=1}^{N_{s}}$. A kernel density estimate (Silverman, 1986) is then used to approximate the likelihood from this sample. Briefly, the general formulation for a kernel density estimate is

$$
L\left(\tilde{T}, C \mid b, A, \mu_{v 1}, \mu_{v 2}, \mu_{w 1}, \mu_{w 2}, t_{0}+t_{\text {rate }}\right) \approx \frac{1}{N_{s}} \sum_{k=1}^{N_{s}} w_{h}\left(\tilde{T}-\tau_{k}\right) \delta_{C}\left(c_{k}\right),
$$

where $\delta_{C}$ is the Kronecker delta function that is 1 when $c_{k}=C$ and 0 otherwise, $w_{h}$ is a scaled weighting function

$$
w_{h}(t)=\frac{1}{h} w\left(\frac{t}{h}\right)
$$

and $w$ is a smooth function that is symmetric about $t=0$ that integrates to 1 . There are numerous options for the specific choice of the weighting function $w$ and bandwidth parameter $h$, both of which affect the accuracy of this approximation. The Epanechnikov kernel (Epanechnikov, 1969) is known to have certain optimal properties (Silverman, 1986). However the Gaussian kernel provides a number of practical benefits, and so we use it here.

This direct method of computing the kernel density estimate proves to be computationally cumbersome. We thus instead use an augmentation of it that uses tools from signal processing to indirectly compute the density estimate much more efficiently (Silverman, 1982; Holmes, 2015). There are a number of rules of thumb for choosing the weighting parameter $h$ (Silverman, 1986). These, however, typically rely on the assumption that data is nearly normal, which is not the case here (see Figure 4c for 
example). We find that with the uniform kernel, the parameters $N_{s}=10,000$ and $h=50 \mathrm{~ms}$ are sufficient to approximate the likelihood. For further details regarding this method and its properties, see (Holmes, 2015).

Using this procedure, for a given set of parameters, the likelihood of each observation and in turn the bulk likelihood of the parameter set can be computed. Note, however, that each switch time represents a separate experimental condition that will augment the likelihood function. Since each block has a separate switch time, this likelihood estimation must be performed for each block separately. This procedure thus requires drawing $N_{s}=10,000$ samples for each of the 15 blocks, for each of the 31 participants. This translates to 4.65 million samples that must be drawn to compute the full likelihood for all participants, for each MCMC chain iteration. Computational efficiency is thus a necessity. We do not detail all the implementation details of this procedure, but note that the likelihood computation is highly parallel. We take advantage of MatLab's built in parallel computing suite to distribute this workload over multiple cores on a desktop to improve efficiency.

Markov Chain Monte Carlo specifics. Following Turner et al. (2013), we fit each of the three hierarchal models in a Bayesian framework using a Differential Evolution Markov Chain Monte Carlo (DE-MCMC) procedure. The purpose of using this procedure rather than a more typical MCMC is the known high degree of correlation between parameters in these LBA type models (Turner et al., 2013) that lead to high rejection rates and poor efficiency. We briefly describe the implementation details specific to this context and refer the reader to Ter Braak (2006), Turner et al. (2013), and Storn and Price (1997) for a more complete discussion.

The basic idea of the DE-MCMC is to make proposals for chain updates informed by the correlation structure of the existing chains. Let $\theta_{i}^{n}$ represent the current state 
(iteration $i$ ) of chain $n$. The DE-MCMC proposal is chosen according to

$$
\theta^{*}=\theta_{i}^{n}+\gamma\left(\theta_{i}^{l}-\theta_{i}^{m}\right)+\epsilon
$$

where $l, m \neq n, \gamma$ controls the proposal jump size, and $\epsilon$ is a randomized symmetry breaking parameter needed to avoid degeneracies. The critical quantities that must be chosen for this procedure are 1) the number of chains $\left(N_{c}\right)$ to be used, 2) a tuning parameter $\gamma$, and 3) the distribution of $\epsilon$. Following Ter Braak (2006), we take $\gamma=2.38 / \sqrt{2 d}$ where $d$ is the dimension of the parameter space being updated, $\epsilon \sim U(-0.001,0.001)$ where $U$ indicates the uniform distribution, and $N_{c}$ is three times the maximum parameter block size, which we discuss next.

Model $1 \mathrm{f}$ is comprised of $31 \times 8=248$ individual level parameters and 16 hyper parameters. For each chain update, these are broken into smaller groups. The hyper parameters are separated into eight groups according to $\left(\mu_{b}, \sigma_{b}\right),\left(\mu_{A}, \sigma_{A}\right),\left(\mu_{V 1}, \sigma_{V 1}\right)$, $\left(\mu_{V 2}, \sigma_{V 2}\right),\left(\mu_{W 1}, \sigma_{W 1}\right),\left(\mu_{W 2}, \sigma_{W 2}\right),\left(\mu_{e r}, \sigma_{e r}\right),\left(\mu_{\text {rate }}, \sigma_{\text {rate }}\right)$. For each chain, each of these blocks is iteratively updated conditioned on the appropriate data as follows:

1. Update $\left(\mu_{b, i}^{n}, \sigma_{b, i}^{n}\right)$ conditioned on $\left\{b_{i-1}^{p, n}\right\}_{p=1}^{P}$,

2. Update $\left(\mu_{A, i}^{n}, \sigma_{A, i}^{n}\right)$ conditioned on $\left\{A_{i,-1}^{p, n}\right\}_{p=1}^{P}$,

3. Update $\left(\mu_{V 1, i}^{n}, \sigma_{V 1, i}^{n}\right)$ conditioned on $\left\{\mu_{v 1, i-1}^{p, n}\right\}_{p=1}^{P}$,

4. Update $\left(\mu_{W 1, i}^{n}, \sigma_{W 1, i}^{n}\right)$ conditioned on $\left\{\mu_{w 1, i-1}^{p, n}\right\}_{p=1}^{P}$,

5. Update $\left(\mu_{V 2, i}^{n}, \sigma_{V 2, i}^{n}\right)$ conditioned on $\left\{\mu_{v 2, i-1}^{p, n}\right\}_{p=1}^{P}$,

6. Update $\left(\mu_{W 2, i}^{n}, \sigma_{W 2, i}^{n}\right)$ conditioned on $\left\{\mu_{w 2, i-1}^{p, n}\right\}_{p=1}^{P}$,

7. Update $\left(\mu_{\text {rate }, i}^{n}, \sigma_{\text {rate }, i}^{n}\right)$ conditioned on $\left\{t_{\text {rate }, i-1}^{p, n}\right\}_{p=1}^{P}$,

8. Update $\left(\mu_{e r, i}^{n}, \sigma_{e r, i}^{n}\right)$ conditioned on $\left\{t_{e r, i-1}^{p, n}\right\}_{p=1}^{P}$,

9a. For each $p=1 \ldots P$, update $\left(b_{i}^{p, n}, A_{i}^{p, n}, \mu_{v 1, i}^{p, n}, \mu_{v 2, i}^{p, n}, t_{e r, i}^{p, n}\right)$ conditioned on the choice and response time data for participant $p$, 
9b. For each $p=1 \ldots P$, update $\left(\mu_{w 1, i}^{p, n}, \mu_{w 2, i}^{p, n}, t_{r a t e, i}^{p, n}\right)$ conditioned on the choice and response time data for participant $p$.

Note that each set of individual level parameters is broken into two groups to reduce the dimensionality of the parameter update and improve performance. These groupings were chosen so that pre (resp. post) switch parameters are updated concurrently, since we expect the correlations between the pre and post switch groups to be weaker than those within the groups. For the symmetric version of the model, steps 4,6 are removed and steps $9 a, 9 b$ are condensed into a single update:

7. For each $p=1 \ldots P$, update $\left(b_{i}^{p, n}, A_{i}^{p, n}, \mu_{v 1, i}^{p, n}, \mu_{v 2, i}^{p, n}, t_{e r, i}^{p, n}, t_{r a t e, i}^{p, n}\right)$ conditioned on the choice and response time data for participant $p$. A similar simplification is made for Model 0. For threshold change models, we use a similar rational in handling the participant level parameter updates. Specifically, the post switch threshold and its associated delay are updated with the post switch parameter block.

The largest grouping of parameters in Model 1 is 6 (for the symmetric version). We thus used $N_{c}=18$ chains for models. Each chain was run for 2500 iterations. After the initial 250 iterations, any outlier chain more than 2 standard deviations from the mean of all chains is reset to the mean. An additional burn-in period of 250 iterations was run (for a total burn-in of 500 iterations) after which all chain data was recorded. Chain mixing was assessed by eye and appears to be sufficient, see Figure B1. This algorithm is coded completely in MATLAB (MathWorks) and the total fit time for the models ranged from $30 \mathrm{~min}$ to 6 hours on a Mac Pro desktop.

Although a detailed investigation of this methodology and its properties is beyond the scope of this exposition (see (Holmes, 2015) for further discussion), we do note that the efficacy of this approximate algorithm was been verified in two ways. First, we verified that it performs well on parameter recovery for synthetic data. Second, we separated the data for all the stationary trials in this experiment and fit the model with both the 
non-parametric kernel density based algorithm as well as an identical MCMC procedure using the analytic likelihood for the LBA model, and found the posteriors to be nearly indistinguishable. 
The Piecewise Linear Ballistic Accumulator 61

\section{Author Note}

WRH and JST were supported by National Science Foundation Grant SES-1556325. AH was supported by an ARC Professorial Fellowship. 
The Piecewise Linear Ballistic Accumulator 62

\section{Footnotes}

1 authors contributed equally

${ }^{2}$ We initially attempted to fit excluded subjects, but found their post switch drift rate posteriors were flat, nearly uniform distributions. We suspect these subjects were inattentive and not being influenced by the change in motion on a sufficient number of trials to fit these parameters. This was supported by a strong correlation between accuracy on the RDM direction-determination task and differential drift rate, as well as by the fact that these participants exhibited a longer rate delay $\left(t_{\text {rate }} \sim 500 \mathrm{~ms}\right)$.

${ }^{3}$ We do not report detailed results for this variant, which corresponds to the full model illustrated in Figure 1, because it was quite unstable, indicating that it was over-parameterised for our data.

${ }^{4}$ We assumed a drift rate of -0.2 that switched to 0.2 (both with a 0.1 between-trial standard deviation and moment-to-moment noise with standard deviation 0.1) with an unbiased start point and a threshold $a=0.1$. The switch occurred at 0.1 s and the mean decision time was $0.2 \mathrm{~s}$. We investigated cases where there was no start-point noise and where it had a uniform distribution with a width of 0.04 
The Piecewise Linear Ballistic Accumulator 63

Table 1

Comparison of Models. DIC comparison of eight variants of the piecewise LBA model. In the case of Models 1-3, the modifiers $s$ and $f$ indicate the type of drift rate switch included (i.e., symmetric or free). In some models, we also allowed for a delay in the change of drift rates and an increase in the threshold (with a potential delay). For each model, a check mark indicates which feature is included in that model. For all model variants, the computed DIC value is provided.

\begin{tabular}{ccccc}
\hline Model Number & Rate Delay & Threshold Increase & Threshold Delay & DIC \\
\hline 0 & $\times$ & $\times$ & $\times$ & 1007 \\
00 & $\times$ & $\times$ & $\times$ & 971 \\
\hline $1 s$ & $\checkmark$ & $\times$ & $\times$ & 951 \\
$2 s$ & $\times$ & $\checkmark$ & $\checkmark$ & 1001 \\
$3 s$ & $\checkmark$ & $\checkmark$ & $\times$ & 937 \\
\hline $1 f$ & $\checkmark$ & $\times$ & $\times$ & 926 \\
$2 f$ & $\times$ & $\checkmark$ & $\checkmark$ & 966 \\
$3 f$ & $\checkmark$ & $\checkmark$ & $\times$ & 922 \\
\hline
\end{tabular}




\section{Table 2}

Correlations between performance and effects. Here we compute correlations between four measures of participant performance on the task with the rate delay and differential effects. The four measures of performance we consider are the accuracy on stationary trials, the hit rate on switch detection trials, the mean response time on stationary trials, and the mean response time on switch trials. Correlations and p-values (in parentheses) are presented. Significant correlation are indicated in bold.

\begin{tabular}{ccc}
\hline & $D^{p}$ & Mean $t_{\text {rate }}$ \\
\hline Stationary Accuracy & $\mathbf{0 . 5 2}(\mathbf{0 . 0 0 3})$ & $-0.21(0.25)$ \\
Detection Hit Rate & $0.17(0.36)$ & $0.15(0.42)$ \\
Mean Stationary RT & $\mathbf{0 . 4 8}(\mathbf{0 . 0 0 7})$ & $-0.03(0.87)$ \\
Mean Post Switch RT & $\mathbf{0 . 4 3 ( \mathbf { 0 . 0 1 5 } )}$ & $-0.027(0.88)$ \\
\hline
\end{tabular}


The Piecewise Linear Ballistic Accumulator 65

\section{Figure Captions}

\section{Figure 1. Piecewise Linear Ballistic Accumulator (PLBA) Model Schematic:}

Evidence for two choice alternatives (i.e., pre-switch correct choice, C1, and the post-switch correct choice, C2, left and right, respectively) accumulate in time starting at randomly and independently sampled initial evidence levels. At the time denoted "Stimulus Change" the experimental stimulus changes, leading to a change in accumulation drift rates after a delay ("Rate Delay"), and a change in threshold after a separate delay ("Threshold Delay"). In the trial depicted, the post-switch correct choice ( $\mathrm{C} 2$ or right) is made (i.e., a correct response corresponding to the changed motion direction).

Figure 2. Behavioral Results: a) For each participant, the proportion of correct responses for stationary (filled circles and boxes) and switch (unfilled circles and boxes) trials before the switch time. Trials were divided based on RT quartiles - q1 (red), q2 (light blue), q3 (dark blue), and q4 (black) and average accuracy within each quantile plotted as a function of average RT within each quantile. The insert shows the median, upper, and lower quartiles of accuracy scores over participants for each RT quartile. b) For each participant, the proportion of correct responses after the switch time for stationary and switch trials by RT quartiles. Note that "correct" was defined relative to the second direction of motion for switch trials c) The median, upper, and lower quartiles of the proportion of correct responses over participants for switch trials and error responses for stationary trials. ${ }^{*} \mathrm{p}<.05$ (Tukey test).

Figure 3. Hyper mean posterior: Posterior distributions for the hyper mean parameters for Model 1f (Panel a) and Model 2f (Panel b). For each panel, the posterior for the relevant hyper mean is presented (e.g., threshold parameters in the first row). The drift rate distributions before and after the switch are shown in the middle panels. 
Horizontal bars indicate the differential drift rate between the accumulators for the pre-switch correct $(\mathrm{C} 1)$ and post-switch correct $(\mathrm{C} 2)$ choices, and the mean value of this differential is quoted in the respective title line.

Figure 4. Quality of Fit for Model Variant 1f Fit: . Panel a) For each participant, the choice probabilities are compute for each of the 15 blocks (4-18) in the direction determination task. Also, the mean of the individual level parameters for each participant are drawn from the posterior, and the predicted choice probabilities are separately computed for each of the 15 blocks, with the switch times taken from the experimental data for that individual participant. The computed and actual choice probabilities are plotted against each other with black (respectively grey) representing the probability of choosing the pre-switch correct choice (C1) (respectively post-switch correct choice, C2). Panels b-d) Comparison of experimental and computed response time distributions for three separate participants. For each participant, data over all 15 blocks is aggregated. Black and grey respectively show the response time distributions for choices $\mathrm{C} 1$ and $\mathrm{C} 2$ respectively. See "Quality of model fit" for further details on the aggregation. Panel e) Comparison of participant quartile accuracy with model predictions on stationary trials. Here, quartiles are computed from the full RT distribution for each participant for only stationary trials. Panel f) Comparison of participant quartile accuracy and model predictions on switch trials. Here, quartiles are derived from switch trials where responses occur after the change of information.

Figure 5. Individual participant parameter correlations: Correlations between lower level parameters for Model 1f, for an individual participant. The lower triangle depicts correlations between the chain values for the given parameters, the diagonals show the posterior in histogram form, and the upper triangle provides the numeric correlations. 
Figure 6. Population distribution estimates: The histograms are based on 100,000 samples, where for each sample a random participant then a random parameter vector from that participant's posterior was chosen and the rate delay (left panel) and rate asymmetry (right panel) measures obtained.

Figure B1. Visual assessment of samples: Values of 9 randomly selected chains for the stated parameter in Model 1f. These results indicate good chain mixing at both the individual and hyper parameter levels. The participant presented was chosen at random. 
Figure 2
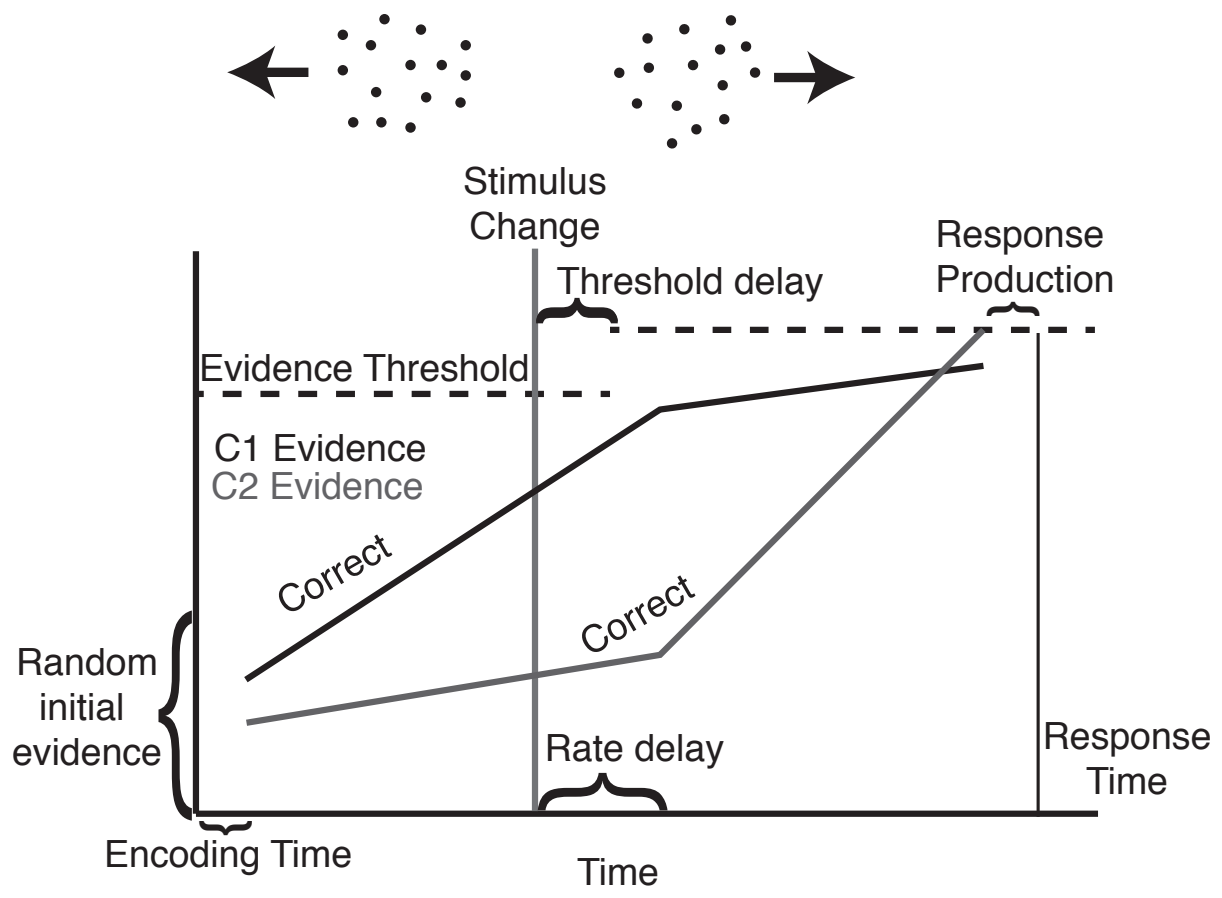
Figure 1

a)

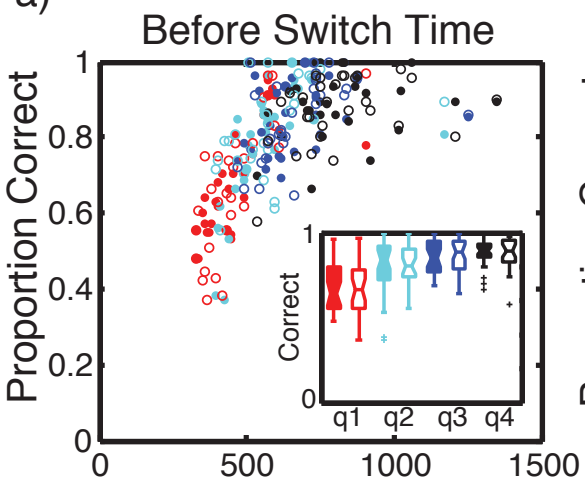

Response Time (ms) b)

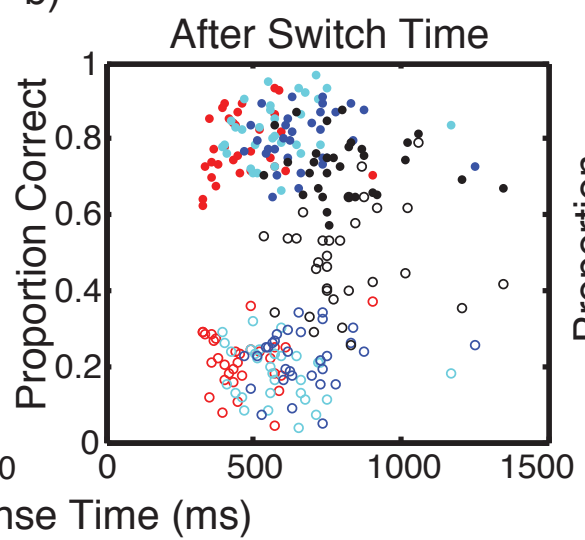

○ Switch c)

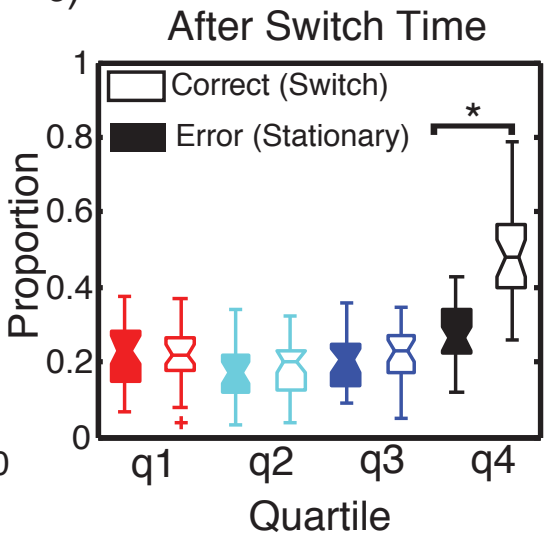

- Stationary

Switch 
Figure 3

a)

Model $1 f$

Threshold, mean $=2.7$

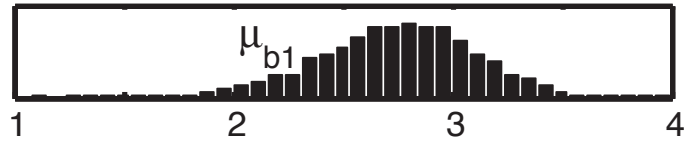

Before, $<$ differential $>=1.3$

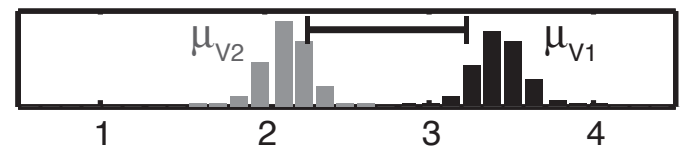

After, $<$ differential $>=2.3$

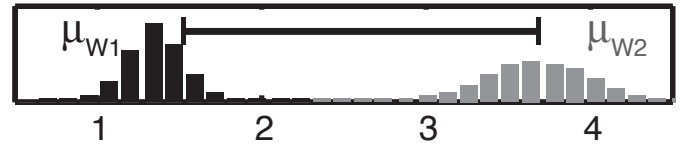

$\mu_{\text {rate }}$, mean $=0.44$

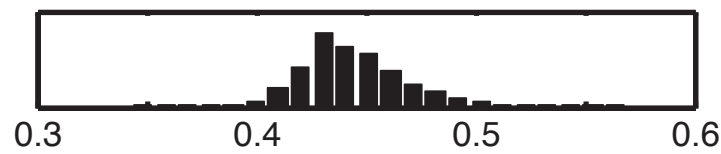

b) Model $2 f$

Threshold, mean $=2.6$

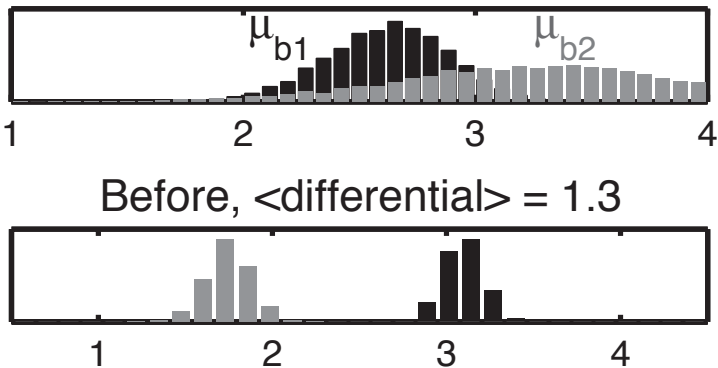

After, $<$ differential $>=0.25$

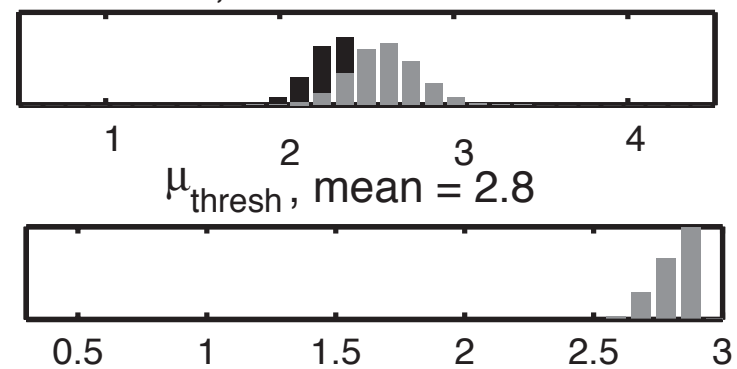


Figure 4

a)

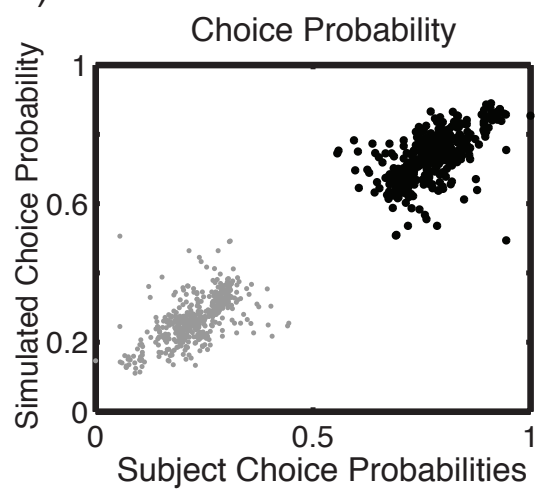

d)

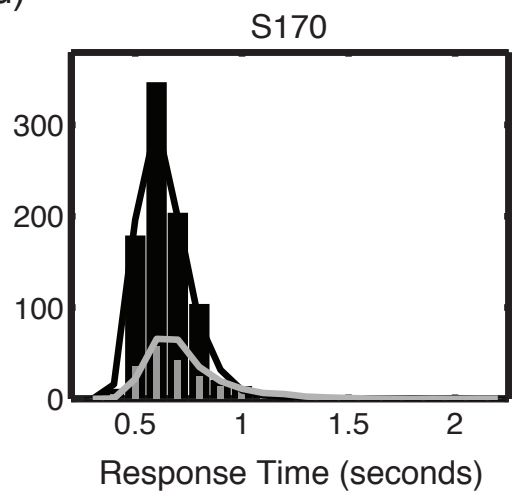

b)

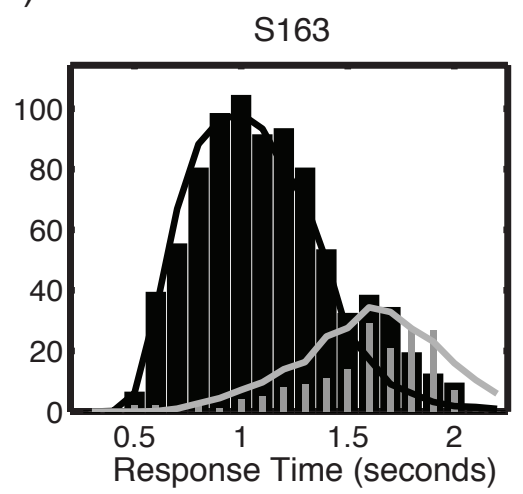

e)

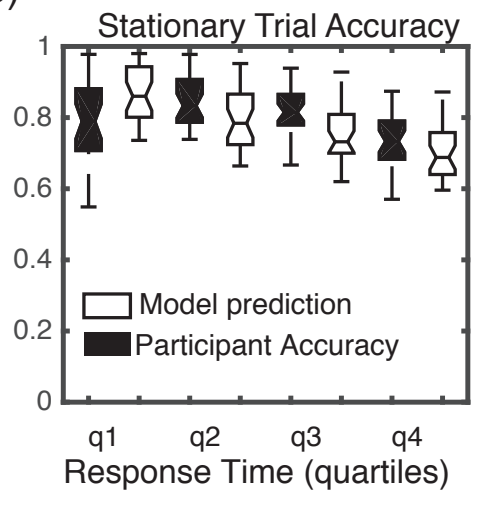

c)

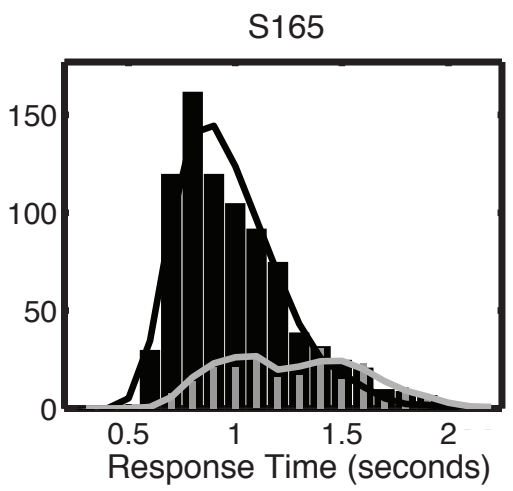

f) Switch Trial Accuracy

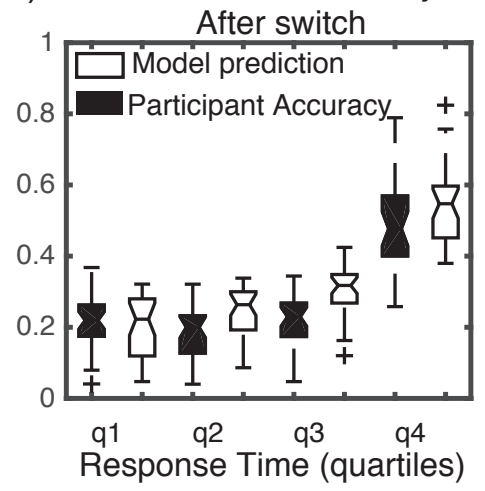


Figure 5

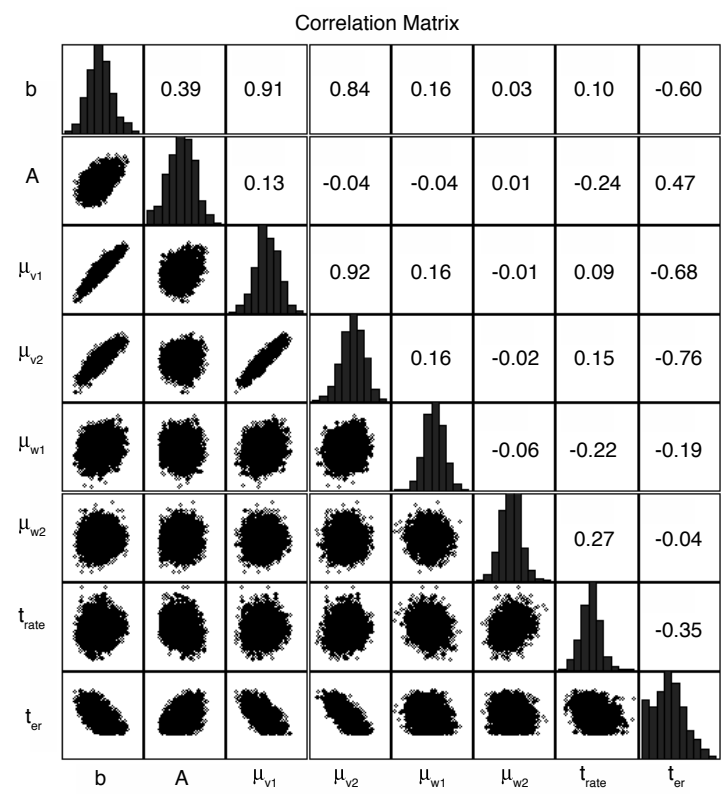


Figure B1
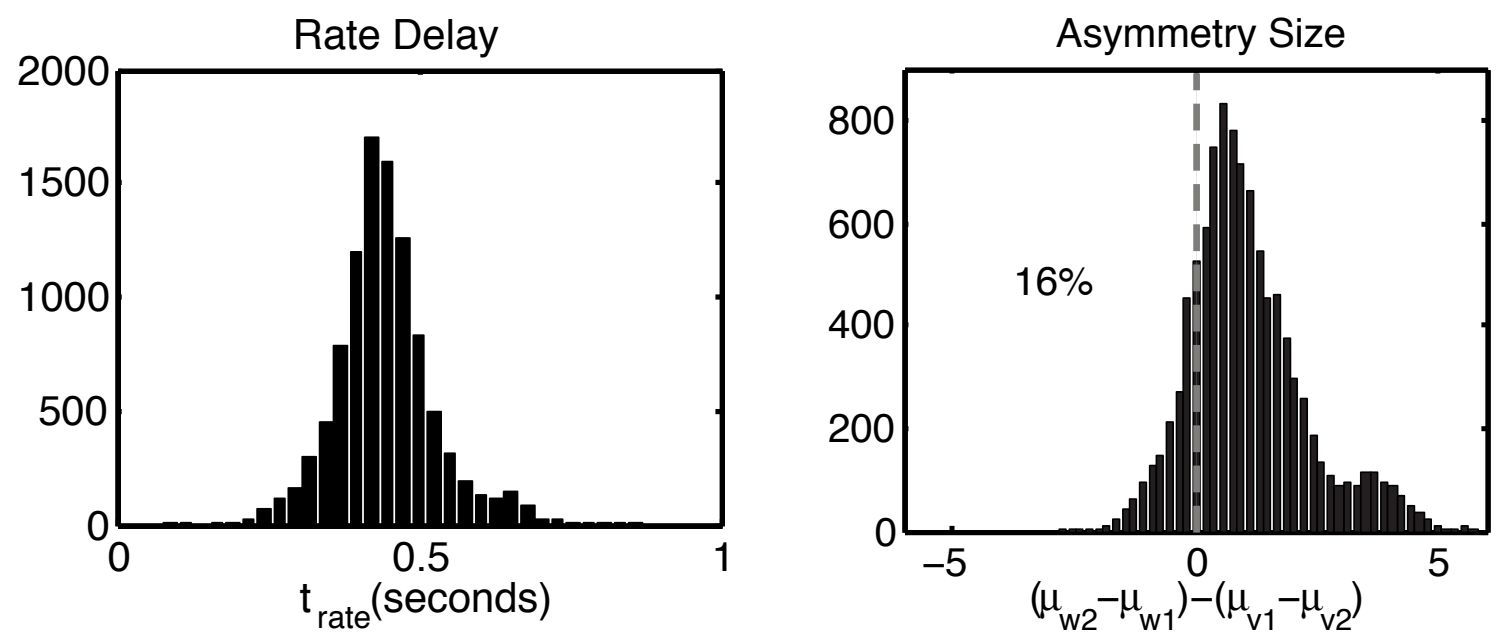
Figure 6

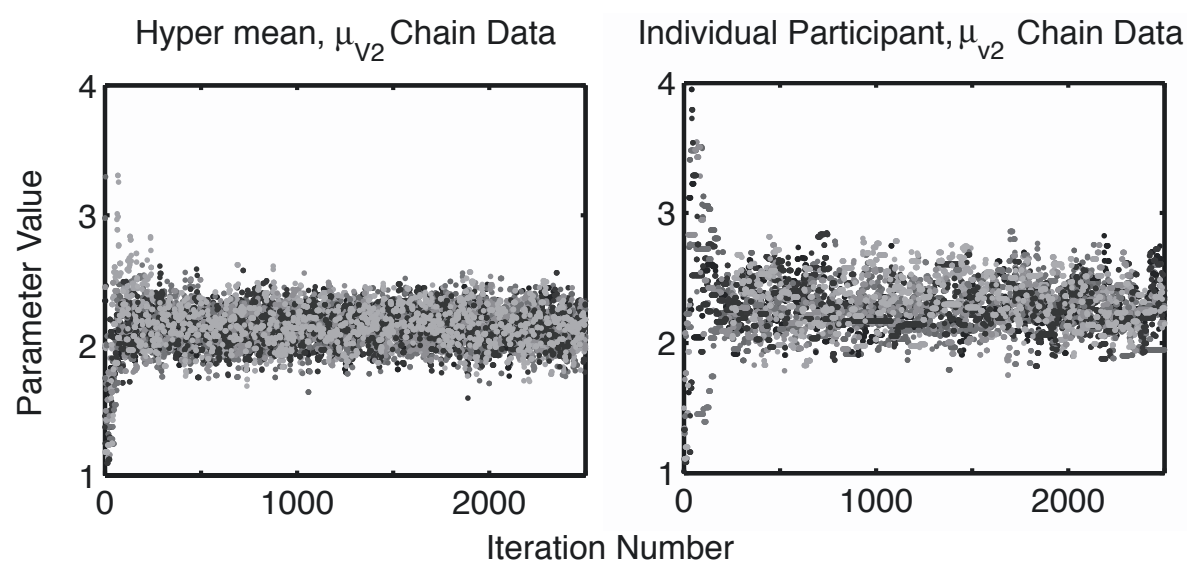

